\title{
Periodic Striped Ground States in Ising Models with Competing Interactions
}

\author{
Alessandro Giuliani ${ }^{1}$, Robert Seiringer ${ }^{2}$ \\ 1 Dipartimento di Matematica e Fisica, Università degli Studi Roma Tre, L.go S. L. Murialdo 1, \\ 00146 Roma, Italy. E-mail: giuliani@mat.uniroma3.it \\ 2 Institute of Science and Technology Austria, Am Campus 1, 3400 Klosterneuburg, Austria.
}

Received: 9 September 2015 / Accepted: 14 March 2016

Published online: 20 June 2016 - (c) The Author(s) 2016. This article is published with open access at Springerlink.com

\begin{abstract}
We consider Ising models in two and three dimensions, with short range ferromagnetic and long range, power-law decaying, antiferromagnetic interactions. We let $J$ be the ratio between the strength of the ferromagnetic to antiferromagnetic interactions. The competition between these two kinds of interactions induces the system to form domains of minus spins in a background of plus spins, or vice versa. If the decay exponent $p$ of the long range interaction is larger than $d+1$, with $d$ the space dimension, this happens for all values of $J$ smaller than a critical value $J_{c}(p)$, beyond which the ground state is homogeneous. In this paper, we give a characterization of the infinite volume ground states of the system, for $p>2 d$ and $J$ in a left neighborhood of $J_{c}(p)$. In particular, we prove that the quasi-one-dimensional states consisting of infinite stripes $(d=2)$ or slabs $(d=3)$, all of the same optimal width and orientation, and alternating magnetization, are infinite volume ground states. Our proof is based on localization bounds combined with reflection positivity.
\end{abstract}

\section{Introduction and Main Results}

The problem of proving the emergence of periodic patterns in systems with competing interactions is ubiquitous in several areas of physics, biology and material science [2], ranging from superconductor physics [12], micromagnetism [10], polymer suspensions [24], martensitic phase transitions [27], quantum Hall systems [14], to metal-oxidesemiconductor field-effect transistors [38], nuclear matter [32], and many others [5,6]. In all these systems, experiments or simulations show evidence for the formation of remarkable patterns in suitable regions of the phase diagram. Examples are stripes, bubbles, zig-zag patterns, and columnar phases. The fundamental understanding of these phenomena is still in a primitive stage, mostly based on variational computations and special assumptions on the structure of the low-energy states. There are just a few special cases where the periodicity of the ground state can be proved from first principles $[13,25,26,40,41]$. 
A particularly interesting and poorly understood phenomenon is that of periodic stripe formation [29,30,37,39]. In a series of papers, this phenomenon was studied in Ising and related models with short range attractive and long range repulsive interactions. The method of block reflection positivity led to rigorous proof of the existence of periodic striped states both in one dimension $[15,17,18]$ and in certain two-dimensional models, including a toy model for martensitic phase transitions [22] and a model of in-plane spins with discrete orientations and dipolar interactions [16]. However, the physically interesting case of out-of-plane spins with dipolar, or dipolar-like, interactions, which is of great importance for the physics of thin magnetic films, eluded any rigorous treatment so far.

In a recent work [20,21], we succeeded in computing the specific ground state energy of such a system, with power-law decaying repulsive interactions and decay exponent $p>2 d$ in $d=2,3$ space dimensions, asymptotically as the ferromagnetic transition line is approached. Our estimates allowed us to prove emergence of periodic stripe order in a suitable asymptotic sense, but they were not strong enough to fully control the ground state structure, or to prove breaking of rotational symmetry in the ground state. In this paper we extended the ideas of [19-21] and prove that periodic striped states of optimal width are exact infinite volume ground states. Moreover, we give a characterization of infinite volume ground states that are invariant under translations by one (for $d=2$ ) or two (for $d=3$ ) independent fixed lattice vectors.

The setting is the following: consider Ising models defined by the formal Hamiltonian

$$
H(\underline{\sigma})=-J \sum_{\langle\mathbf{x}, \mathbf{y}\rangle}\left(\sigma_{\mathbf{x}} \sigma_{\mathbf{y}}-1\right)+\sum_{\{\mathbf{x}, \mathbf{y}\}} \frac{\left(\sigma_{\mathbf{x}} \sigma_{\mathbf{y}}-1\right)}{|\mathbf{x}-\mathbf{y}|^{p}}
$$

where $\underline{\sigma} \in\{ \pm 1\}^{\mathbb{Z}^{d}}, d \geq 2$. The first sum in (1.1) ranges over nearest neighbor pairs in $\mathbb{Z}^{d}$, while the second ranges over pairs of distinct sites in $\mathbb{Z}^{d}$. For different values of the exponent $p$, this model is used to describe the effects of frustration induced in magnetic films by the presence of dipolar interactions $(p=3)$ or in charged systems by the presence of an unscreened Coulomb interaction $(p=1)$, as well as many other frustrated systems [1,3-9, 11,23,28,29,31,33-36,39,42].

In this paper, we choose the exponent $p$ to satisfy the constraint $p>2 d$. As discussed in a previous work [20,21], if $J>J_{c}$, with

$$
J_{c}:=\sum_{y_{1}>0, \mathbf{y}^{\perp} \in \mathbb{Z}^{d-1}} \frac{y_{1}}{\left(y_{1}^{2}+\left|\mathbf{y}^{\perp}\right|^{2}\right)^{p / 2}},
$$

then there are exactly two ground states, $\sigma_{\mathbf{x}} \equiv+1 \forall \mathbf{x} \in \mathbb{Z}^{d}$, and $\sigma_{\mathbf{x}} \equiv-1 \forall \mathbf{x} \in \mathbb{Z}^{d}$. For $J<J_{c}$, the ground state is not uniform, and for $J$ close to $J_{c}$ it was conjectured to be a periodic striped configuration, i.e., a quasi-one-dimensional periodic configuration of the form $\left(\underline{\sigma}^{(h)}\right)_{\mathbf{x}}=\operatorname{sign}\left(\sin \left(\pi\left(x_{1}+1 / 2\right) / h\right)\right)$, or translations or rotations thereof, for a suitable stripe width $h \in \mathbb{N}$. In this paper, we prove this conjecture, and characterize the set of infinite volume ground states invariant under translations generated by $d-1$ lattice vectors.

For simplicity, we restrict the discussion to $d=2$ and $p>4$ from now on. Similar considerations are valid in $d=3$ and $p>6$ (or, in fact, for any $d \geq 2$ with $p>2 d$ ). In Appendix A we explain how to adapt the proof to dimension three and higher. Let $e_{\mathrm{s}}(h)$ be the energy per site of $\underline{\sigma}^{(h)}$ computed via (1.1). We let $h^{*}=\operatorname{argmin}_{h \in \mathbb{N}} e_{\mathrm{S}}(h)$, which 
is uniquely defined for almost all ${ }^{1}$ choices of $J$. We denote by $\underline{\sigma}^{*}=\underline{\sigma}^{\left(h^{*}\right)} \in\{ \pm 1\}^{\mathbb{Z}^{2}}$, and we call it an optimal periodic striped configuration. Other $4 h^{*}-1$ optimal periodic striped configurations are obtained from $\sigma^{*}$ via translations and rotations.

In order to state our main result, we also need to introduce the following notions: the configuration $\underline{s} \in\{ \pm 1\}^{\mathbb{Z}^{2}}$ is called an infinite volume ground state if it is energetically stable against compactly supported perturbations, that is, for any finite $X \subset \mathbb{Z}^{2}$,

$$
H_{X}\left(\underline{\sigma}_{X} \mid \underline{s}\right)-H_{X}\left(\underline{s}_{X} \mid \underline{s}\right) \geq 0, \quad \forall \underline{\sigma}_{X} \in\{ \pm 1\}^{X}
$$

where $\underline{s}_{X}$ is the restriction of $\underline{s}$ to $X$, and

$$
\begin{aligned}
H_{X}\left(\underline{\sigma}_{X} \mid \underline{s}\right)= & -J \sum_{\substack{\langle\mathbf{x}, \mathbf{y}\rangle: \\
\mathbf{x}, \mathbf{y} \in X}}\left(\sigma_{\mathbf{x}} \sigma_{\mathbf{y}}-1\right)+\sum_{\substack{\{\mathbf{x}, \mathbf{y}\}: \\
\mathbf{x}, \mathbf{y} \in X}} \frac{\left(\sigma_{\mathbf{x}} \sigma_{\mathbf{y}}-1\right)}{|\mathbf{x}-\mathbf{y}|^{p}} \\
& -J \sum_{\substack{\mathbf{x} \in X, \mathbf{y} \in X^{c}: \\
|\mathbf{x}-\mathbf{y}|=1}}\left(\sigma_{\mathbf{x}} s_{\mathbf{y}}-1\right)+\sum_{\mathbf{x} \in X, \mathbf{y} \in X^{c}} \frac{\left(\sigma_{\mathbf{x}} s_{\mathbf{y}}-1\right)}{|\mathbf{x}-\mathbf{y}|^{p}} .
\end{aligned}
$$

We shall say that two infinite volume ground states are equivalent, if they only differ on a finite set. The equivalence class of a given infinite volume ground state is called a sector. A sector is trivial, if it contains only one element. In terms of these notions, our main result can be summarized as follows.

Theorem 1. There exists $\varepsilon>0$ such that, if $J_{c}-\varepsilon<J<J_{c}$, then the optimal periodic striped configurations are infinite volume ground states, and their sectors are trivial.

This result is a corollary of a quantitative lower bound on the energy of spin configurations, which will be formulated in Theorem 3 below, after having introduced a few more definitions. Our quantitative bounds also allow us to characterize the infinite volume ground states that are invariant under translation by a vector $\mathbf{n} \in \mathbb{Z}^{2}$.

Theorem 2. Under the same conditions as Theorem 1, any infinite volume ground state $\underline{s}$ that is invariant under translation by a vector $\mathbf{n}=\left(n_{1}, n_{2}\right) \in \mathbb{Z}^{2}$ is characterized by the following property: there exists an "interface" of finite width, of the form $\mathcal{I}_{k_{1}, k_{2}}(\mathbf{n})=$ $\left\{\mathbf{x} \in \mathbb{Z}^{2}: \mathbf{x} \cdot \mathbf{n}_{\perp} \in\left[k_{1}, k_{2}\right]\right\}$, where $\mathbf{n}_{\perp}=\left(-n_{2}, n_{1}\right)$ and $k_{1}<k_{2}$ are two integers, such that $\underline{s}$ coincides with two of the optimal striped configurations on the two infinite components of $\left(\mathcal{I}_{k_{1}, k_{2}}(\mathbf{n})\right)^{c}$.

Let us now introduce a few more definitions, which are required for the formulation of our quantitative lower bound on the energy of a generic spin configuration.

\subsection{On Good and Bad.}

1.1.1. Contours and Corners. Given $\underline{\sigma} \in\{ \pm 1\}^{\mathbb{Z}^{2}}$, we let $\Delta=\left\{\mathbf{x} \in \mathbb{Z}^{2}:(\underline{\sigma})_{\mathbf{x}}=-1\right\}$, and $\Gamma(\Delta)$ be its boundary, i.e., the union of bonds of the dual lattice $\left(\mathbb{Z}^{2}\right)^{*}$ separating a point $\mathbf{x} \in \Delta$ from a point $\mathbf{y} \in \Delta^{c}$. At every vertex of $\Gamma(\Delta) \cap\left(\mathbb{Z}^{2}\right)^{*}$, there can be either two or four sides meeting. In the case of four sides, we deform the polygon slightly by "chopping off" the edge from the squares containing a - spin; see Fig. 1.

\footnotetext{
1 There are exceptional values of $J$ for which $e_{\mathrm{S}}(h)$ has two minimizers, $h^{*}$ and $h^{*}+1$. For more detailed comments about the nature of ground states at the exceptional values of J, see the end of Sect. 1.
} 
(a)

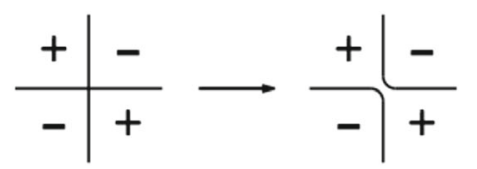

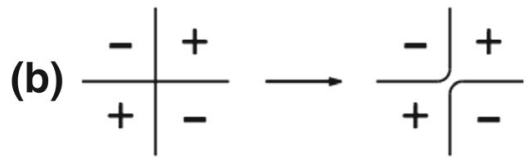

Fig. 1. In the case that four sides of the closed polygon $\Gamma(\Delta)$ meet at a vertex $v$, we slightly deform $\Gamma(\Delta)$ so that the two squares containing a - spin become disconnected from the vertex itself. a The situation where the minus spins are located at NE and SW of $v$, before and after the "chopping". b The situation where the minus spins are located at NW and SE of $v$, before and after the "chopping"

After the chopping, $\Gamma(\Delta)$ splits into disconnected polygons $\Gamma_{1}, \Gamma_{2}, \ldots$, which are called contours. The points where two orthogonal portions of a contour meet are called corners. The sites of the dual lattice where a non-trivial chopping operation took place correspond to two corners. We denote by $N_{c}\left(\Gamma_{i}\right)$ the number of corners of $\Gamma_{i}$, and $N_{c}(\Delta)=\sum_{i} N_{c}\left(\Gamma_{i}\right)$. Note that, if $\underline{\sigma}$ is a compactly supported perturbation of $\underline{\sigma}^{*}$, that is, if the set $\left\{\mathbf{x} \in \mathbb{Z}^{2}:(\underline{\sigma})_{\mathbf{x}} \neq\left(\underline{\sigma}^{*}\right)_{\mathbf{x}}\right\}$ is finite, then $N_{c}(\Delta)<+\infty$.

1.1.2. Tiles. Given an integer $\ell$, we pave $\mathbb{Z}^{2}$ with tiles of side $\ell$ and, given a tile $T$, we denote by $\Gamma_{T}(\Delta)$ the union of the bonds in $\Gamma(\Delta)$ that separate a point in $\Delta \cap T$ from a point in $\Delta^{c}$. Note that this convention assigns uniquely every bond in $\Gamma(\Delta)$ to one of the tiles. The connected components of $\Gamma_{T}(\Delta)$ are contained in the contours $\Gamma_{1}, \ldots$, and are denoted by $\Gamma_{T, i}, i=1, \ldots, r_{T}$. Given a maximal straight portion of $\Gamma_{T, i}$, we assign to each of its two endpoints a "number of corners", which can be either $1 / 2$ or 0 , depending on whether or not the given endpoint coincides with one of the corners in $\cup_{j \geq 1} \Gamma_{j}$. This assignment induces a notion of "number of corners in the tile $T$ ", to be denoted by $n_{c}(T)$, which is the sum of the number of corners of all the endpoints of the straight portions of $\Gamma_{T, i}$, with $i=1, \ldots, r_{T}$. Note that $n_{c}(T)$ can be either integer or half-integer, and $\sum_{T} n_{c}(T)=N_{c}(\Delta)$.

1.1.3. Bad Tiles and Good Regions. We now identify the tiles $T_{i}$ such that either $n_{c}\left(T_{i}\right)>0$, or they contain a square of side $\ell / 5$ completely contained in $\Delta$ or in $\Delta^{c}$, to be called hole. We call these tiles bad ${ }^{2}$, and we let $\mathcal{N}_{B}$ be their number. For later convenience, we also let $\mathcal{N}_{B}^{\text {hole }}$ be the number of bad tiles containing a hole. The connected components of the complement of $\cup_{i=1}^{\mathcal{N}_{B}} T_{i}$, are denoted by $G_{i}, i=1, \ldots, \mathcal{N}_{G}$, and are called the good regions. By construction, any of these connected components contains portions of contours in $\Gamma(\Delta)$ that are all straight with the same orientation, and have no corners. We denote by $\Gamma_{G_{i}}$ the union of contours in $\Gamma(\Delta)$ contained in $G_{i}$. If the elements of $\Gamma_{G_{i}}$ are all vertical, we will say that $G_{i}$ is vertically striped, and horizontally striped otherwise. Consider a good region $G_{i}$ that is vertically (resp. horizontally) striped. We say that $R$ is a "rectangular portion of stripe" in $G_{i}$, if $R$ is a rectangle completely contained in $\Delta$ or in $\Delta^{c}$, with its two vertical (resp. horizontal) boundaries both belonging to $\Gamma_{G_{i}}$. We also define the distance between its two vertical (resp. horizontal) boundaries to be the width of $R$. Finally, we let $A_{h}\left(G_{i}\right)$ denote the area of the union of all the rectangular portions of stripes of width $h$ in $G_{i}$. Note that, if $\underline{\sigma}$ is a compactly supported perturbation of $\underline{\sigma}^{*}$, then $\sum_{i=1}^{\mathcal{N}_{G}} A_{h}\left(G_{i}\right)$ is finite, $\forall h \neq h^{*}$.

We are now in the position of stating our quantitative lower bound on the energy $H_{X}\left(\underline{s}_{X} \mid \underline{\sigma}^{*}\right)$ of a spin configuration $\underline{s}$ with $\underline{\sigma}^{*}$ boundary conditions.

\footnotetext{
${ }^{2}$ We shall choose $\ell$ large compared to the optimal stripe width $h^{*}$, which explains why we expect a hole to be energetically unfavorable, hence bad.
} 
Theorem 3. There exist positive constants $C_{0}, C_{1}, \varepsilon$ such that, if $J_{c}-\varepsilon<J<J_{c}$ and $C_{0} h^{*} \leq \ell \leq\left(C_{0}\left(J_{c}-J\right)\right)^{-1}$, then for every $\underline{s} \in\{ \pm 1\}^{\mathbb{Z}^{2}}$ and every finite set $X \subset \mathbb{Z}^{2}$,

$$
\begin{aligned}
H_{X}\left(\underline{s}_{X} \mid \underline{\sigma}^{*}\right) \geq & H_{X}\left(\underline{\sigma}_{X}^{*} \mid \underline{\sigma}^{*}\right)+C_{1}\left(N_{c}+\left(J_{c}-J\right)^{\frac{p-2}{p-3}} \ell^{2} \mathcal{N}_{B}^{\text {hole }}\right) \\
& +\frac{1}{2} \sum_{h \neq h^{*}} \sum_{i=1}^{\mathcal{N}_{G}}\left(e_{\mathrm{s}}(h)-e_{\mathrm{s}}\left(h^{*}\right)\right) A_{h}\left(G_{i}\right)
\end{aligned}
$$

where $N_{c}, \mathcal{N}_{B}^{\text {hole }}$, and $G_{i}$ are, respectively, the number of corners, the number of bad tiles containing a hole, and the good regions, associated with the infinite spin configuration $\underline{\sigma}=\left(\underline{s}_{X}, \underline{\sigma}_{X^{c}}^{*}\right)$ coinciding with $\underline{s}_{X}$ on $X$ and with $\underline{\sigma}^{*}$ on $X^{c}$, defined via tiling with squares of side length $\ell$ as described above.

Remark. Since $h^{*} \sim\left(J_{c}-J\right)^{-1 /(p-3)} \ll\left(J_{c}-J\right)^{-1}$ for small $J_{c}-J$ (compare with Eq. (2.30) below), the condition on $\ell$ can be fulfilled for $J$ close to $J_{c}$. Note also that $e_{\mathrm{S}}\left(h^{*}\right) \sim\left(J_{c}-J\right)^{(p-2) /(p-3)}$ for small $J_{c}-J$, which agrees with the factor multiplying $\mathcal{N}_{B}^{\text {hole }}$ in the second term on the right side of (1.5).

Remark. The prefactor $1 / 2$ in the second line of (1.5) can be replaced by any number less than 1 , at the expense of modifying the constants $C_{0}$ and $\varepsilon$.

Theorem 3 implies, in particular, that $\sigma^{*}$ is an infinite volume ground state, and that every state $\underline{\sigma}$ that is a compactly supported perturbation of it, is not a ground state, simply because any such state necessarily has corners and, therefore, by (1.5), it has strictly larger energy than $\underline{\sigma}^{*}$. This immediately implies Theorem 1 .

In order to see that also Theorem 2 is a consequence of Theorem 3, consider an infinite volume ground state $\underline{s}$ that is invariant under translations by an integer vector $\mathbf{n}$. Let $\Lambda \subset \mathbb{Z}^{2}$ be a square box of side $L$, and note that the energy price for changing the boundary conditions from $\underline{s}$ to $\underline{\sigma}^{*}$ scales like the boundary, that is

$$
\left|H_{\Lambda}\left(\underline{\sigma}_{\Lambda} \mid \underline{s}\right)-H_{\Lambda}\left(\underline{\sigma}_{\Lambda} \mid \underline{\sigma}^{*}\right)\right| \leq 2\left(J+J_{c}\right)|\partial \Lambda|
$$

for any $\underline{\sigma}_{\Lambda}$. Using this inequality and the very definition of infinite volume ground state, we have

$$
\begin{aligned}
& H_{\Lambda}\left(\underline{\sigma}_{\Lambda}^{*} \mid \underline{\sigma}^{*}\right)+2\left(J+J_{c}\right)|\partial \Lambda| \\
& \quad \geq H_{\Lambda}\left(\underline{\sigma}_{\Lambda}^{*} \mid \underline{s}\right) \geq H_{\Lambda}\left(\underline{s}_{\Lambda} \mid \underline{s}\right) \\
& \quad \geq H_{\Lambda}\left(\underline{s}_{\Lambda} \mid \underline{\sigma}^{*}\right)-2\left(J+J_{c}\right)|\partial \Lambda| .
\end{aligned}
$$

Now we apply Theorem 3, thus obtaining

$$
\begin{aligned}
& C_{1}\left(N_{c}+\left(J_{c}-J\right)^{\frac{p-2}{p-3}} \ell^{2} \mathcal{N}_{B}^{\text {hole }}\right)+\frac{1}{2} \sum_{h \neq h^{*}} \sum_{i=1}^{\mathcal{N}_{G}}\left(e_{\mathrm{S}}(h)-e_{\mathrm{s}}\left(h^{*}\right)\right) A_{h}\left(G_{i}\right) \\
& \leq 4\left(J+J_{c}\right)|\partial \Lambda|,
\end{aligned}
$$

where $N_{c}, \mathcal{N}_{B}^{\text {hole }}$ and $G_{i}$ refer to the configuration $\left(\underline{s}_{\Lambda}, \underline{\sigma}_{\Lambda^{c}}^{*}\right)$. In particular, $N_{c}$ is bounded by (const.) $L$. Since every corner not at the boundary of $\Lambda$ is repeated with period $\mathbf{n}$, there can be at most a finite number of them (modulo translations by $\mathbf{n}$ ) independently of $L$. This means that these corners are all contained in a finite strip $\mathcal{I}_{k_{1}, k_{2}}(\mathbf{n})$, as claimed 
in Theorem 2, with $k_{1}, k_{2}$ independent of $L$. Similarly, we can argue that the holes and the stripes of width different from $h^{*}$ are all contained in a finite strip $\mathcal{I}_{k_{1}, k_{2}}(\mathbf{n})$. This concludes the proof of Theorem 2, in the case that $h^{*}$ is unique. As observed above, there are exceptional values of $J$ for which $e_{\mathrm{s}}(h)$ has two minimizers, $h^{*}$ and $h^{*}+1$. In these cases, the discussion above leaves open the possibility that on one of the connected components of $\left(\mathcal{I}_{k_{1}, k_{2}}(\mathbf{n})\right)^{c}$ the stripes are not all of the same width. However, this cannot be the case: by proceeding as in [15, Section III.D], one can prove that each pair of neighboring stripes of widths $h^{*}, h^{*}+1$ gives an extra positive contribution to the energy per unit stripe length. Therefore, pairs of stripes of different widths are all contained in a finite strip $\mathcal{I}_{k_{1}, k_{2}}(\mathbf{n})$, and Theorem 2 follows.

The rest of the paper is devoted to the proof of Theorem 3.

\section{Proof of Theorem 3}

The proof of Theorem 3 is divided into several steps, and uses many notations and ideas introduced in [20,21], which will be recalled here. In reading the proof, it may be useful to keep in mind that the goal is to derive lower bounds for the energy of the "Peierls contours", i.e., of the connected components of the union of the bad tiles, and to show that we gain in energy by erasing the contours, as in the standard Peierls argument. The difficulty lies in the fact that the energy of these contours, as well as the one of the good regions, is non-local, due to the power-law nature of the repulsive interaction, and a priori it depends on the geometric shape of the surrounding good and bad regions. In order to get a useful, local, bound on the contour energies, it is convenient to devise a localization procedure, which allows us to replace the original non-local Hamiltonian by a sum of local energies, supported on the bad and good regions, respectively. The localization procedure must be performed carefully in order to minimize the resulting boundary error (which is proportional to the boundary between the good and bad regions). For this purpose, we also need to get refined bounds on the localized energies of the bad and good regions, and to show that the excess energy associated with the Peierls contours is large enough to compensate the aforementioned boundary error. Compared to our previous paper [20,21], a key technical novelty is our improved lower bound on the energy of the good regions, which may have complicated geometrical shapes. The crucial estimate is summarized in Lemma 1 below. A similar estimate already appeared in [20,21], but only for rectangular good regions, which was a severe restriction.

In more detail, the proof is organized as follows:

- In Sect. 2.1 we reduce to plus boundary conditions, which allows us to use the droplet formulation for the energy as in [20,21], see (2.3) below. Rewriting the energy in terms of droplet self-energies and droplet-droplet interactions is advantageous, in that it provides us with a good localization procedure, with boundary errors that are much smaller than those obtained by localizing directly the original spin Hamiltonian.

- In Sect. 2.2 we describe the localization procedure and, for the purpose of a lower bound, we replace the original Hamiltonian by a sum of local energies, each supported on the bad tiles or on the good regions, respectively.

- In Sect. 2.3 we state our main bounds on the localized energies, and show that they easily imply Theorem 3 .

- In Sect. 2.4 (resp. 2.5) we give the proof of the lower bound on the local energy of the good regions (resp. bad tiles). 
Before entering the proof, we recall some notation and relevant scales: we let $\tau=$ $2\left(J-J_{c}\right)$, which is assumed to be negative and small, in absolute value. The energy per site $e_{\mathrm{s}}(h)$ of a periodic striped configuration of stripe width $h$ is equal to $\tau h^{-1}+A_{p} h^{-p+2}$, for a suitable constant $A_{p}>0$, up to a negligible errors for large $h$ (see (2.30) below for the exact formula). This readily implies that the optimal energy, i.e., the minimum of $e_{\mathrm{S}}(h)$, is negative and of the order $|\tau|^{(p-2) /(p-3)}$, and the optimal stripe width $h^{*}=$ $\operatorname{argmin} e_{\mathrm{S}}(h)$ is of the order $|\tau|^{-1 /(p-3)}$. In the following, we will take the tile side length $\ell$ entering the definition of bad and good regions to be much larger than $h^{*}$, in order to guarantee that good regions contain many stripes (which is required for making the boundary errors introduced in the localization procedure negligible compared to the bulk energy), and smaller than order $|\tau|^{-1}$, in order to guarantee that the excess energy of the bad tiles is proportional to the number of corners (cf. Eqs. (2.38)-(2.41) below).

2.1. Reduction to Plus Boundary Conditions. The $\sigma^{*}$ boundary conditions, while natural in the perspective of proving uniqueness of the ground state, are not particularly convenient for using the droplet representation of [20,21]. However, a few simple algebraic manipulations allow us to reduce to the same boundary conditions of $[20,21]$ (that is, plus boundary conditions) in a suitable enlarged box. To see this, rewrite $H_{X}\left(\underline{\sigma}_{X} \mid \underline{\sigma}^{*}\right)$ as

$$
H_{X}\left(\underline{\sigma}_{X} \mid \underline{\sigma}^{*}\right)=H_{X}\left(\underline{\sigma}_{X}^{*} \mid \underline{\sigma}^{*}\right)+\lim _{\Lambda \nearrow \mathbb{Z}^{2}}\left[H_{\Lambda}^{\text {per }}\left(\underline{\sigma}_{X}, \underline{\sigma}_{\Lambda \mid X}^{*}\right)-H_{\Lambda}^{\text {per }}\left(\underline{\sigma}_{\Lambda}^{*}\right)\right]
$$

where $\Lambda$ is a square box of side $L$, which we choose to be divisible by $2 h^{*},\left(\underline{\sigma}_{X}, \underline{\sigma}_{\Lambda \backslash X}^{*}\right)$ is the configuration on $\Lambda$ whose restriction to $X \subset \Lambda$ (resp. $\Lambda \backslash X$ ) coincides with $\underline{\sigma}_{X}$ (resp. $\left.\underline{\sigma}_{\Lambda \backslash X}^{*}\right)$, and $H_{\Lambda}^{\text {per }}\left(\underline{\sigma}_{\Lambda}\right)$ is the Hamiltonian with periodic, rather than $\underline{\sigma}^{*}$, boundary conditions.

Now, $H_{\Lambda}^{\text {per }}\left(\underline{\sigma}_{\Lambda}^{*}\right)=e_{\mathrm{S}}\left(h^{*}\right)|\Lambda|$ and $H_{\Lambda}^{\text {per }}\left(\underline{\sigma}_{X}, \underline{\sigma}_{\Lambda \mid X}^{*}\right)$ can be further rewritten in terms of a Hamiltonian with plus boundary conditions:

$$
H_{\Lambda}^{\mathrm{per}}\left(\underline{\sigma}_{X}, \underline{\sigma}_{\Lambda \backslash X}^{*}\right)=\lim _{M \rightarrow \infty} \frac{1}{M^{2}} H_{\Lambda_{M}}^{+}\left(\left(\underline{\sigma}_{X}, \underline{\sigma}_{\Lambda \backslash X}^{*}\right)^{M^{2}}\right)
$$

where $\Lambda_{M}$ is a square box of side $L M$, to be thought of as the union of $M^{2}$ copies of $\Lambda$, and $\left(\underline{\sigma}_{X}, \underline{\sigma}_{\Lambda \backslash X}^{*}\right)^{M^{2}}$ is a symbol for the configuration on $\Lambda_{M}$ obtained by juxtaposing $M^{2}$ copies of $\left(\underline{\sigma}_{X}, \underline{\sigma}_{\Lambda \backslash X}^{*}\right)$, one in each of the copies of $\Lambda$. Moreover, $H_{X}^{+}\left(\underline{\sigma}_{X}\right)=H_{X}\left(\underline{\sigma}_{X} \mid \underline{\sigma}^{+}\right)$ indicates the Hamiltonian with plus boundary conditions (here $\underline{\sigma}^{+}$is the uniform infinite spin configuration consisting of plus spins everywhere). Finally, for later reference, we introduce the shorthand $\underline{u}_{\Lambda_{M}}$ for the spin configuration $\left(\underline{\sigma}_{X}, \underline{\sigma}_{\Lambda \backslash X}^{*}\right)^{M^{2}}$ on $\Lambda_{M}$, and $\underline{u}$ for the infinite one coinciding with $\underline{u}_{\Lambda_{M}}$ on $\Lambda_{M}$ and with $\underline{\sigma}^{+}$on the complement. From now on we shall consider $\underline{u}_{\Lambda_{M}}$ and $\underline{u}$ fixed once and for all.

2.2. Localization. We now re-express $H_{\Lambda_{M}}^{+}\left(\underline{u}_{\Lambda_{M}}\right)$ in terms of the droplets representation introduced in [20,21]. Using the notation introduced in Sect. 1.1.1, we let $\Delta$ be the region of minus spins associated with $\underline{u}, \Gamma(\Delta)$ its boundary, and $\Gamma_{1}, \ldots, \Gamma_{r}$ the corresponding contours. We also denote by $\mathfrak{G}(\Delta)$ the collection of contours, $\mathfrak{G}(\Delta)=\left\{\Gamma_{1}, \ldots, \Gamma_{r}\right\}$. Note that $\Delta, \Gamma(\Delta), \mathfrak{G}(\Delta)$ and $r$ are finite, because of the plus boundary conditions. As in $[20,21]$, we denote by $\delta_{i}$ the maximal connected components of $\Delta$, and by $\mathcal{D}(\Delta)$ 

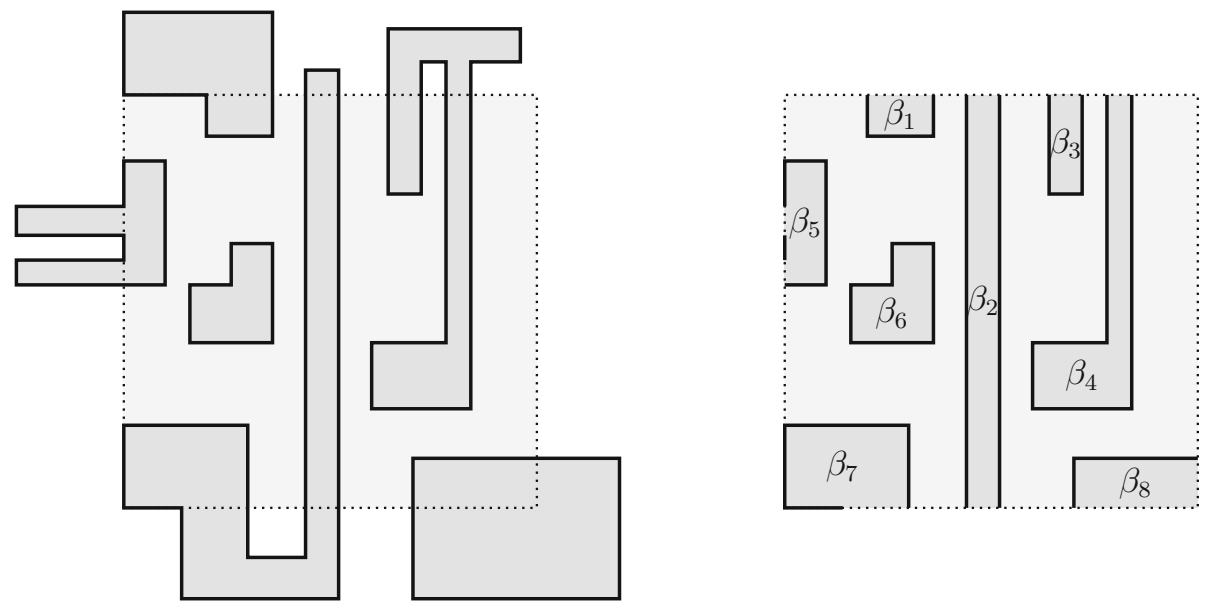

Fig. 2. Left a square region $Q$ (in light grey, with dotted boundary) and the droplets having non-zero intersection with it. Right the corresponding bubble configuration $\mathcal{B}_{Q}$ after localization in $Q$. Note that a single droplet can give rise after localization to more than one bubble: e.g., $\beta_{2}$ and $\beta_{7}$ originate from the same droplet, and similarly for $\beta_{3}$ and $\beta_{4}$. Note also that the contour of a bubble does not necessarily coincide with the boundary of its droplet: in general, it is contained in it, and may even be disconnected (as in the case of $\beta_{5}$, whose contour consists of two disconnected portions)

their collection. Given $\delta \in \mathcal{D}(\Delta)$, we also let $\Gamma(\delta)$ be the boundary of $\delta$, and $N_{c}(\delta)$ its number of corners. In terms of these notations, we can re-express the energy of $\underline{u}_{\Lambda_{M}}$ as

$$
H_{\Lambda_{M}}^{+}\left(\underline{u}_{\Lambda_{M}}\right)=2 J \sum_{\Gamma \in \mathfrak{G}(\Delta)}|\Gamma|+\sum_{\delta \in \mathcal{D}(\Delta)} U(\delta)+\frac{1}{2} \sum_{\substack{\delta, \delta^{\prime} \in \mathcal{D}(\Delta) \\ \delta \neq \delta^{\prime}}} W\left(\delta, \delta^{\prime}\right),
$$

where

$$
U(\delta):=-2 \sum_{\mathbf{x} \in \delta} \sum_{\mathbf{y} \in \mathbb{Z}^{2} \mid \delta} \frac{1}{|\mathbf{x}-\mathbf{y}|^{p}}, \quad W\left(\delta, \delta^{\prime}\right):=4 \sum_{\mathbf{x} \in \delta} \sum_{\mathbf{y} \in \delta^{\prime}} \frac{1}{|\mathbf{x}-\mathbf{y}|^{p}}
$$

Let us consider the partition $\mathcal{P}$ of $\Lambda_{M}$ defined by the bad tiles and good regions of $\Lambda_{M}$, in the sense of Sect. 1.1.3: $\mathcal{P}=\left\{T_{i}\right\}_{i=1}^{\mathcal{N}_{B}} \cup\left\{G_{i}\right\}_{i=1}^{\mathcal{N}_{G}}$. We now localize the energy in the elements of $\mathcal{P}$, by proceeding as in [20,21, Section 3]. More precisely, we derive a lower bound on the energy $H_{\Lambda_{M}}^{+}\left(\underline{u}_{\Lambda_{M}}\right)$ in the form of a sum of local energies $E_{Q}\left(\mathcal{B}_{Q}\right)$, each depending only on the "bubble configuration" $\mathcal{B}_{Q}$ within the region $Q \in \mathcal{P}$. The notion of bubble configuration was introduced in [20,21, Section 3] and is recalled here: given $Q \in \mathcal{P}$ and $\delta \in \mathcal{D}$, we denote by $\Gamma_{Q}(\delta)$ the portion of $\Gamma(\delta)$ belonging to $Q$. Moreover, if $\delta_{Q}=\delta \cap Q$, we define $\bar{\delta}_{Q}^{(1)}, \ldots, \bar{\delta}_{Q}^{\left(m_{Q}(\delta)\right)}$ to be the maximal connected components of $\delta_{Q}$, and $\bar{\Gamma}_{Q}^{(1)}, \ldots, \bar{\Gamma}_{Q}^{\left(m_{Q}(\delta)\right)}$ to be the portions of $\Gamma_{Q}(\delta)$ belonging to the boundary of $\bar{\delta}_{Q}^{(1)}, \ldots, \bar{\delta}_{Q}^{\left(m_{Q}(\delta)\right)}$, respectively. We shall refer to the pair $\left(\bar{\delta}_{Q}^{(i)}, \bar{\Gamma}_{Q}^{(i)}\right)$ as to a bubble in $Q$ originating from $\delta$. We shall indicate by $\mathcal{B}_{Q}(\delta)$ the set of bubbles in $Q$ originating from $\delta$, and by $\mathcal{B}_{Q}=\cup_{\delta \in \mathcal{D}} \mathcal{B}_{Q}(\delta)$ the total set of bubbles in $Q$ (see Fig. 2). 
Given a bubble $\beta=\left(\delta_{\beta}, \Gamma_{\beta}\right) \in \mathcal{B}_{Q}$, we also define its localized self-energy as

$$
u_{Q}(\beta)=-\sum_{b \in \Gamma_{\beta}} \sum_{\mathbf{n} \neq \mathbf{0}} \frac{\min \left\{\left|n_{1}\right|, d_{b}^{Q}\left(\delta_{\beta}\right)\right\}}{|\mathbf{n}|^{p}},
$$

where $d_{b}^{Q}\left(\delta_{\beta}\right)$ is the distance between $b$ and the bond $b^{\prime} \in \Gamma_{\beta}$ facing it ${ }^{3}$ in $\beta$, if there is such a bond, and is infinite, if $b$ faces the boundary of $Q$. In terms of these notions, our localization bound takes the following form:

$$
H_{\Lambda_{M}}^{+}\left(\underline{u}_{\Lambda_{M}}\right) \geq \sum_{i=1}^{\mathcal{N}_{B}} E_{T_{i}}\left(\mathcal{B}_{T_{i}}\right)+\sum_{i=1}^{\mathcal{N}_{G}} E_{G_{i}}\left(\mathcal{B}_{G_{i}}\right),
$$

where

$$
\begin{aligned}
& E_{T_{i}}\left(\mathcal{B}_{T_{i}}\right)=\sum_{\beta \in \mathcal{B}_{T_{i}}}\left[2 J\left|\Gamma_{\beta}\right|+u_{T_{i}}(\beta)\right]+\frac{1}{2} \sum_{\substack{\beta, \beta^{\prime} \in \mathcal{B}_{T_{i}}, \beta \neq \beta^{\prime}}} W\left(\delta_{\beta}, \delta_{\beta^{\prime}}\right)+2^{1-p / 2} n_{c}\left(T_{i}\right), \\
& E_{G_{i}}\left(\mathcal{B}_{G_{i}}\right)=\sum_{\beta \in \mathcal{B}_{G_{i}}}\left[2 J\left|\Gamma_{\beta}\right|+u_{G_{i}}(\beta)\right]+\frac{1}{2} \sum_{\substack{\beta, \beta^{\prime} \in \mathcal{B}_{G_{i}}, \beta \neq \beta^{\prime}}}^{*} W\left(\delta_{\beta}, \delta_{\beta^{\prime}}\right),
\end{aligned}
$$

and the $*$ on the last sum indicates the following constraint: if the bubbles in $G_{i}$ are all vertical (resp. horizontal) we only sum over pairs of bubbles that do not overlap after arbitrary translations in the vertical (resp. horizontal) direction.

Remark. The right side of (2.6) should be thought of as a first step towards the derivation of a Peierls-like bound on the "contours", consisting of the connected components of the union of the bad tiles. Compared with the original Hamiltonian, the right side of (2.6) has the advantage of being a sum of independent contributions, localized in the bad and good regions, respectively, without long-range interactions between the regions. The local energy functionals have a structure very similar to the original droplet Hamiltonian, with an explicit excitation energy associated to the corners (in the case of $E_{T_{i}}\left(\mathcal{B}_{T_{i}}\right)$ ). The bound is sharp, in the sense that equality holds in (2.6) if $\mathcal{N}_{B}=0$. The energy of the good regions will be bounded from below by $e_{\mathrm{S}}\left(h^{*}\right)$ times the total area of the good regions, up to a boundary error scaling like $|\tau| \sum_{i=1}^{\mathcal{N}_{G}}\left|\partial G_{i}\right|$, while every bad tile will be shown to have an excess energy (compared to the reference energy $e_{\mathrm{S}}\left(h^{*}\right) \ell^{2}$ ) larger than $\max \left\{1, \ell^{2}|\tau|^{(p-2) /(p-3)}\right\}$, which is enough to compensate the boundary error. The total excess energy of every contour plays the role of its Peierls energy.

Proof of (2.6). We start from (2.3). The goal is to bound it from below by a sum of terms, each of which is localized in an element $Q$ of $\mathcal{P}$. The first term on the right side of (2.3)

3 The notion of "bond facing $b$ in $\beta$ " is defined as follows. Let us suppose for definiteness that $b \in \Gamma_{\beta}$ is vertical and that it separates a point $\mathbf{x}_{b} \in \delta_{\beta}$ on its immediate right from a point $\mathbf{y}_{b}=\mathbf{x}_{b}-(1,0) \notin \delta_{\beta}$ on its immediate left. We say that $b$ faces $b^{\prime} \in \Gamma_{\beta}$, and vice versa, if: (i) $b^{\prime}$ is vertical; (ii) $b^{\prime}$ separates a point $\mathbf{x}_{b^{\prime}} \in \delta_{\beta}$ on its immediate left from a point $\mathbf{y}_{b^{\prime}}=\mathbf{x}_{b^{\prime}}+(1,0) \notin \delta_{\beta}$ on its immediate right; (iii) the points $\mathbf{x}_{b}$ and $\mathbf{x}_{b^{\prime}}$ are at the same height, i.e., $\left[\mathbf{x}_{b}\right]_{2}=\left[\mathbf{x}_{b^{\prime}}\right]_{2}$, and all the points on the same row between them belong to $\delta_{\beta}$ : in other words, $\mathbf{x}_{b}+(j, 0) \in \delta_{\beta}$, for all $j=0, \ldots,\left[\mathbf{x}_{b^{\prime}}\right]_{1}-\left[\mathbf{x}_{b}\right]_{1}$. An analogous definition is valid for horizontal bonds. If $b$ does not face any $b^{\prime} \in \Gamma_{\beta}$, we say that $b$ faces the boundary of $Q$. 
is already local, i.e., it can be rewritten exactly as $2 J \sum_{Q \in \mathcal{P}} \sum_{\beta \in \mathcal{B}_{Q}}\left|\Gamma_{\beta}\right|$, which leads to the corresponding terms in (2.7) and (2.8). The interaction $W\left(\delta, \delta^{\prime}\right)$ on the right side of (2.3) can be rewritten as

$$
W\left(\delta, \delta^{\prime}\right)=\sum_{Q, Q^{\prime} \in \mathcal{P}} \sum_{\substack{\beta \in \mathcal{B}_{Q}(\delta) \\ \beta^{\prime} \in \mathcal{B}_{Q^{\prime}}\left(\delta^{\prime}\right)}} W\left(\delta_{\beta}, \delta_{\beta^{\prime}}\right)
$$

which is bounded from below by dropping the terms with $Q \neq Q^{\prime}$ (recall that the interaction is positive), so that

$$
\frac{1}{2} \sum_{\substack{\delta, \delta^{\prime} \in \mathcal{D}(\Delta) \\ \delta \neq \delta^{\prime}}} W\left(\delta, \delta^{\prime}\right) \geq \frac{1}{2} \sum_{\substack{Q \in \mathcal{P} \\ \beta, \beta^{\prime} \in \mathcal{B}_{Q} \\ \beta \neq \beta^{\prime}}}^{* *} W\left(\delta_{\beta}, \delta_{\beta^{\prime}}\right)
$$

and the $* *$ on the sum indicates the constraint that the two droplets $\delta, \delta^{\prime}$ in $\mathcal{D}(\Delta)$, which $\delta_{\beta}, \delta_{\beta^{\prime}}$ belong to, are different from each other, $\delta \neq \delta^{\prime}$.

Regarding the second term on the right side of (2.3), we bound it from below by using [20,21, Eq. (2.9)], that is

$$
U(\delta) \geq-\sum_{b \in \Gamma(\delta)} \sum_{\mathbf{n} \neq \mathbf{0}} \frac{\min \left\{\left|n_{1}\right|, d_{b}(\delta)\right\}}{|\mathbf{n}|^{p}}+2^{1-\frac{p}{2}} N_{c}(\delta)+4 \sum_{\{\mathbf{x}, \mathbf{y}\} \in \mathcal{P}(\delta)} \frac{1}{|\mathbf{x}-\mathbf{y}|^{p}}
$$

where $d_{b}(\delta)$ is the distance between $b$ and the bond $b^{\prime}$ facing it in $\delta$, and $\mathcal{P}(\delta)$ is the set of unordered pairs of distinct sites in $\delta$ such that both $\mathcal{C}_{\mathbf{x} \rightarrow \mathbf{y}}^{h v}$ and $\mathcal{C}_{\mathbf{x} \rightarrow \mathbf{y}}^{v h}$ cross at least two bonds of $\Gamma(\delta)$. Here $\mathcal{C}_{\mathbf{x} \rightarrow \mathbf{y}}^{h v}$ is the path on the lattice that goes from $\mathbf{x}$ to $\mathbf{y}$ consisting of two segments, the first horizontal and the second vertical. Similarly, $\mathcal{C}_{\mathbf{x} \rightarrow \mathbf{y}}^{v h}$ is the path on the lattice that goes from $\mathbf{x}$ to $\mathbf{y}$ consisting of two segments, the first vertical and the second horizontal (note that the two paths can coincide, in the case that $x_{i}=y_{i}$ for some $i \in\{1,2\})$.

The first term on the right side of (2.10) can be bounded from below as

$$
-\sum_{b \in \Gamma(\delta)} \sum_{\mathbf{n} \neq \mathbf{0}} \frac{\min \left\{\left|n_{1}\right|, d_{b}(\delta)\right\}}{|\mathbf{n}|^{p}} \geq \sum_{Q \in \mathcal{P}} \sum_{\beta \in \mathcal{B}_{Q}(\delta)} u_{Q}(\beta)
$$

which, after summation over $\delta$, leads to the terms $\sum_{\beta \in \mathcal{B}_{Q}} u_{Q}(\beta)$ in (2.7) and (2.8). The second term on the right side of (2.10) is local and, after summation over $\delta$, can be rewritten as $2^{1-\frac{p}{2}} \sum_{i=1}^{\mathcal{N}_{B}} n_{c}\left(T_{i}\right)$ (recall that the good regions have no corners), which leads to the last term on the right side of (2.7). Moreover, the sum over $\delta$ of the last term on the right side of (2.10) can be bounded from below by an expression similar to the right side of (2.9), namely

$$
\sum_{\delta \in \mathcal{D}(\Delta)} 4 \sum_{\{\mathbf{x}, \mathbf{y}\} \in \mathcal{P}(\delta)} \frac{1}{|\mathbf{x}-\mathbf{y}|^{p}} \geq \frac{1}{2} \sum_{\substack{Q \in \mathcal{P} \\ \beta, ~}}^{\dagger} \sum_{\substack{\beta^{\prime} \in \mathcal{B}_{Q} \\ \beta \neq \beta^{\prime}}}^{\dagger} W\left(\delta_{\beta}, \delta_{\beta^{\prime}}\right)
$$


where the $\dagger$ on the sum indicates the constraint that $\delta_{\beta}, \delta_{\beta^{\prime}}$ belong to the same droplet $\delta \in \mathcal{D}(\Delta)$, and all the pairs of points $(\mathbf{x}, \mathbf{y})$ in $\delta_{\beta} \times \delta_{\beta^{\prime}}$ are such that $\{\mathbf{x}, \mathbf{y}\} \in \mathcal{P}(\delta)$. Combining the right sides of (2.9) and (2.12) we obtain

$$
\frac{1}{2} \sum_{Q \in \mathcal{P}} \sum_{\substack{\beta, \beta^{\prime} \in \mathcal{B}_{Q} \\ \beta \neq \beta^{\prime}}}^{\dagger \dagger} W\left(\delta_{\beta}, \delta_{\beta^{\prime}}\right),
$$

where the $\dagger \dagger$ on the sum indicates the constraint that: either $\delta_{\beta}, \delta_{\beta^{\prime}}$ belong to different droplets in $\mathcal{D}(\Delta)$, or, if they belong to the same droplet in $\delta$, they are such that all pairs of points $(\mathbf{x}, \mathbf{y})$ in $\delta_{\beta} \times \delta_{\beta^{\prime}}$ are in $\mathcal{P}(\delta)$. Finally, note that: if $Q$ is a bad tile (which is a convex region), then the constraint $\dagger \dagger$ is automatically realized (i.e., it can be dropped), which leads to the second term on the right side of (2.7); if $Q$ is a good region, then the constraint $\dagger \dagger$ is easily seen to be weaker than the one indicated by $*$ in (2.8), which leads to the last term on the right side of (2.8).

2.3. Lower Bounds on the Localized Energies. In this subsection we state the key lower bounds on the localized energies in the bad and good regions, and prove that they imply Theorem 3. Recall the definitions of $\mathcal{N}_{B}, \mathcal{N}_{B}^{\text {hole }}$ and $A_{h}\left(G_{i}\right)$ given in Sect. 1.1.3. Recall also that $\ell$ is the side length of the tiles, which enters the definition of the partition $\mathcal{P}$.

Lemma 1. There exist positive constants $c_{0}, c_{1}$ and $\varepsilon$ such that, if $-\varepsilon<\tau<0$ and $\ell \geq c_{0} h^{*}$, then the energy $E_{G}$ of any good region $G \in \mathcal{P}$ satisfies

$$
E_{G}\left(\mathcal{B}_{G}\right) \geq e_{\mathrm{S}}\left(h^{*}\right)|G|-c_{1}|\tau||\partial G|+\frac{1}{2} \sum_{h \neq h^{*}}\left(e_{\mathrm{S}}(h)-e_{\mathrm{S}}\left(h^{*}\right)\right) A_{h}(G) .
$$

Lemma 2. There exist positive constants $c_{0}, c_{2}$ and $\varepsilon$ such that, if $-\varepsilon<\tau<0$ and $c_{0} h^{*} \leq \ell \leq\left(c_{0}|\tau|\right)^{-1}$, then the energy $E_{T}$ of any bad tile $T \in \mathcal{P}$ satisfies

$$
E_{T}\left(\mathcal{B}_{T}\right) \geq \ell^{2} e_{\mathrm{S}}\left(h^{*}\right)+c_{2}\left[n_{c}(T)+|\tau|^{(p-2) /(p-3)} \ell^{2} \chi_{\text {hole }}(T)\right],
$$

where $\chi_{\text {hole }}(T)$ is equal to 1 if $T$ contains a hole, and 0 otherwise.

Lemma 1 is one of the main technical novelties of this paper, and its proof is described in detail in the Sect. 2.4. The boundary error on the right side of (2.13), proportional to $\tau\left|\partial G_{i}\right|$, is "optimal", in the sense that its value is of the same order as the one obtained by comparing the energy of an optimal striped configuration in a rectangular region with open boundary conditions with that in the same region with periodic boundary conditions. The proof of Lemma 2 is simpler than the one of Lemma 1, it is an extension of the bounds worked out in [20,21], and its proof is postponed to Sect. 2.5.

Combining the two lemmas, we can easily derive Theorem 3 . The key observation is that every portion of the boundary $\partial G_{i}$ of a good region $G_{i} \in \mathcal{P}$ is adjacent to a bad tile, so that the boundary error $-c_{1}|\tau| \ell$ associated with every "elementary portion" of $\partial G_{i}$ is compensated by the excess energy of the adjacent bad tile. More precisely, using the fact that every portion of $\cup_{i} \partial G_{i}$ is adjacent to a bad tile, we get that $\sum_{i=1}^{\mathcal{N}_{G}}\left|\partial G_{i}\right| \leq 4 \ell \mathcal{N}_{B}$. 
Therefore, using also the fact that every bad tile either has a positive number of corners (at least 1/2) or a hole, plugging (2.13) and (2.14) into (2.6), we obtain

$$
\begin{aligned}
H_{\Lambda_{M}}^{+}\left(\underline{u}_{\Lambda_{M}}\right) \geq & e_{\mathrm{S}}\left(h^{*}\right)\left|\Lambda_{M}\right|+\frac{c_{2}}{2}\left(N_{c}(\Delta)+|\tau|^{(p-2) /(p-3)} \ell^{2} \mathcal{N}_{B}^{\text {hole }}\right) \\
& +\frac{1}{2} \sum_{h \neq h^{*}} \sum_{i=1}^{\mathcal{N}_{G}}\left(e_{\mathrm{S}}(h)-e_{\mathrm{S}}\left(h^{*}\right)\right) A_{h}\left(G_{i}\right) \\
& +\mathcal{N}_{B}\left(\frac{c_{2}}{2} \min \left\{\frac{1}{2},|\tau|^{\frac{p-2}{p-3}} \ell^{2}\right\}-4 c_{1}|\tau| \ell\right) .
\end{aligned}
$$

Now, if

$$
\frac{8 c_{1}}{c_{2}}|\tau|^{-1 /(p-3)}<\ell<\frac{c_{2}}{16 c_{1}}|\tau|^{-1},
$$

then the expression in parentheses that multiplies $\mathcal{N}_{B}$ is positive, and we can drop it for the purpose of a lower bound. Via the use of (2.1) and (2.2), we thus arrive at (1.5).

\subsection{Proof of Lemma 1.}

2.4.1. Deforming the Good Regions. We now discuss the proof of Lemma 1. Let us consider a good region $G \in \mathcal{P}$, and let us assume without loss of generality that it only contains vertical stripes. We first want to slightly deform the domain $G$ and correspondingly change the bubble configuration within, in order to make all the bubbles rectangular: here we call "rectangular" a bubble $\beta=\left(\delta_{\beta}, \Gamma_{\beta}\right)$ such that $\delta_{\beta}$ is a rectangle and $\Gamma_{\beta}$ is the union of its two vertical sides. Note that, in general, not all the bubbles in $G$ are rectangular, due to boundary effects (in fact, the boundary can partially "cut" a portion of the rectangle, without disconnecting it, see e.g. the droplets $\delta_{1}$ and $\delta_{2}$ in Fig. 3).

In order to describe the deformation of $G$, think of the vertical boundary of $G$ as a union of segments $S_{i}$ of length $\ell$, induced by the tiling described in Sects. 1.1.2 and 1.1.3. By construction, every boundary segment $S_{i}$ faces a portion $\tilde{S}_{i}$ of length $\ell$ of the boundary of a rectangular bubble in $\mathcal{B}_{G}$ that is closer than $2 \ell / 5$ to $S_{i}$ itself. We now deform the boundary $\partial G$ continuously, by moving the segments $S_{i}$ towards the interior of $G$, in such a way that they coincide with $\tilde{S}_{i}$; see Fig. 4 .

In this way we increase the boundary of $G$ by at most $|\partial G|$ itself, and we increase the energy by at most $|\tau| \cdot|\partial G|$, which is acceptable for our purposes. We shall denote by $G^{\prime}$ the new region obtained from $G$ by the deformations we just described, and the new bubble configuration $\mathcal{B}_{G^{\prime}}$. We have

$$
E_{G}\left(\mathcal{B}_{G}\right) \geq E_{G^{\prime}}\left(\mathcal{B}_{G^{\prime}}\right)-|\tau| \cdot|\partial G|
$$

as just argued. 


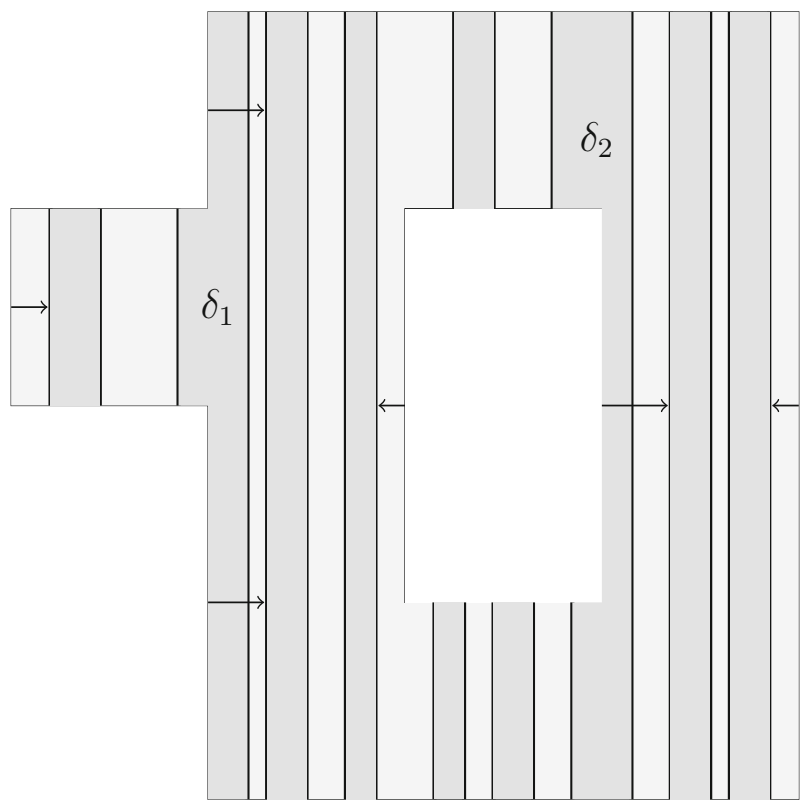

Fig. 3. A good region with its bubble configuration. The regions in dark grey are droplets, while those in light grey are their complement. Note that not all of the droplets are rectangular, due to boundary effects. E.g., the droplets $\delta_{1}$ and $\delta_{2}$ are not rectangular. The arrows indicate the direction in which the vertical boundary segments move under the deformation described in the text

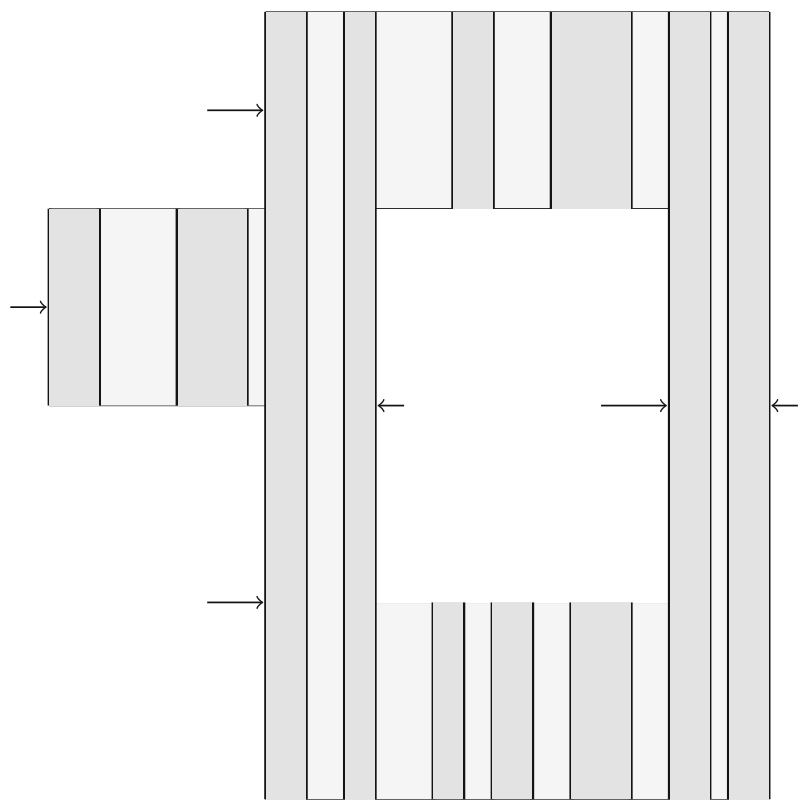

Fig. 4. The deformed good region corresponding to the good region of Fig. 3 , after the moves of the vertical boundary segments indicated by the arrows. Note that after the deformation all the droplets are rectangular, and all the connected components of the complement of the droplets (the connected light grey regions) are rectangular as well 


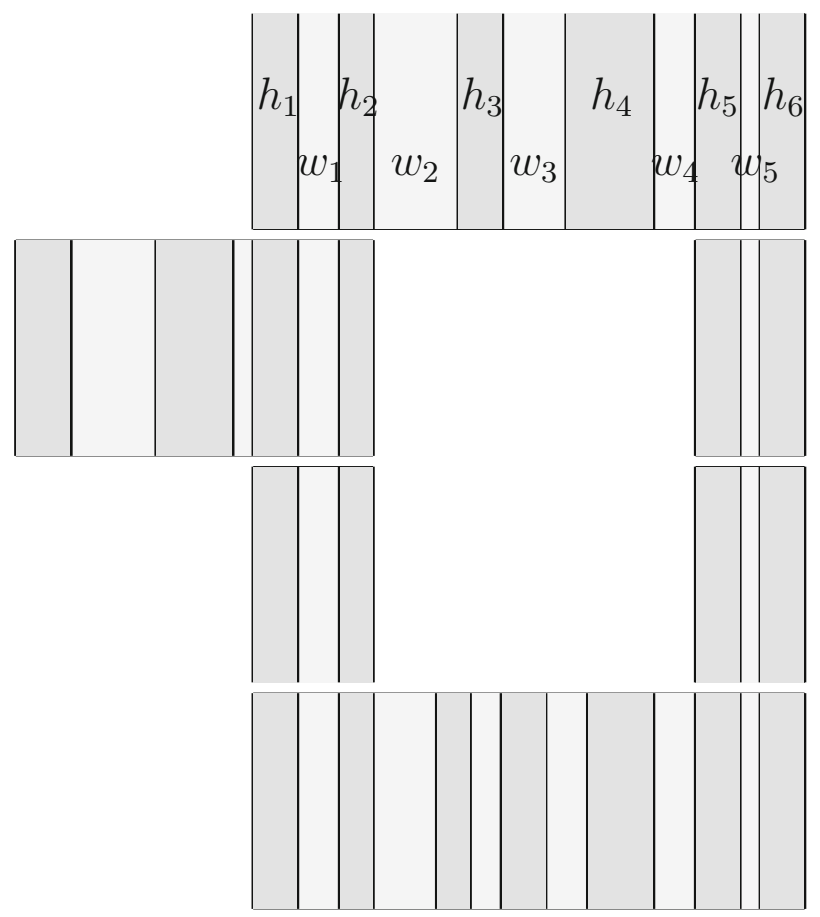

Fig. 5. The six slices corresponding to the deformed good region of Fig. 4. For ease of visualization, the slices are drawn slightly detached from each other. As an example, in the first slice we attached the labels $h_{i}$ and $w_{i}$ indicating the widths of the stripes (dark grey regions) and of the rectangular regions separating two subsequent stripes from each other (light grey regions)

2.4.2. Slicing and Bounding the Energy of the Good Region. The deformed region $G^{\prime}$ obtained in the previous step is a union of connected horizontal slices $g_{j}$ of height $\ell$, with $j=1, \ldots, N_{g}$, as shown in Fig. 5. To each slice we associate a sequence of integers $\left(h_{1}, w_{1}, \ldots, w_{n-1}, h_{n}\right)$, where $n$ is the number of bubbles in $\mathcal{B}_{G^{\prime}}$ intersecting the slice, $h_{1}, h_{2}, \ldots, h_{n}$ are their widths, ordered from left to right, and $w_{1}, w_{2}, \ldots$ are the spacings between the first and second bubbles, second and third, etc.

We also denote by $s_{i}$, with $i=1, \ldots, N_{s}$, the maximal connected segments in the intersection between the horizontal boundary of $G^{\prime}$ and the boundaries of the droplets in $\mathcal{B}_{G^{\prime}}$ (note that the boundary of a rectangular bubble does not coincide with its contour: rather, it consists of four segments, two horizontal and two vertical). Note that segments come in pairs: one can say that two segments form a pair if they belong to the boundary of the same droplet. Moreover, to each segment $s_{j}$ we associate its length $h\left(s_{j}\right)$ and two spacings $w_{1}\left(s_{j}\right), w_{2}\left(s_{j}\right)$, which are the horizontal distances from the next droplets ("next" by following the boundary of $G^{\prime}$ ) to the left and to the right of $s_{j}$. Note that if $s_{j}$ touches a corner of $\partial G^{\prime}$, say on its right side, then there may not be any droplet to its right: by following the boundary one may find that the next segment to the right could actually have the same horizontal coordinates, in which case we will assign the value $+\infty$ to $w_{2}\left(s_{j}\right)$ (and similarly for $w_{1}\left(s_{j}\right)$ in the case of the next segment on the left); see Fig. 6.

The key ingredient for the proof of Lemma 1 is the following. 


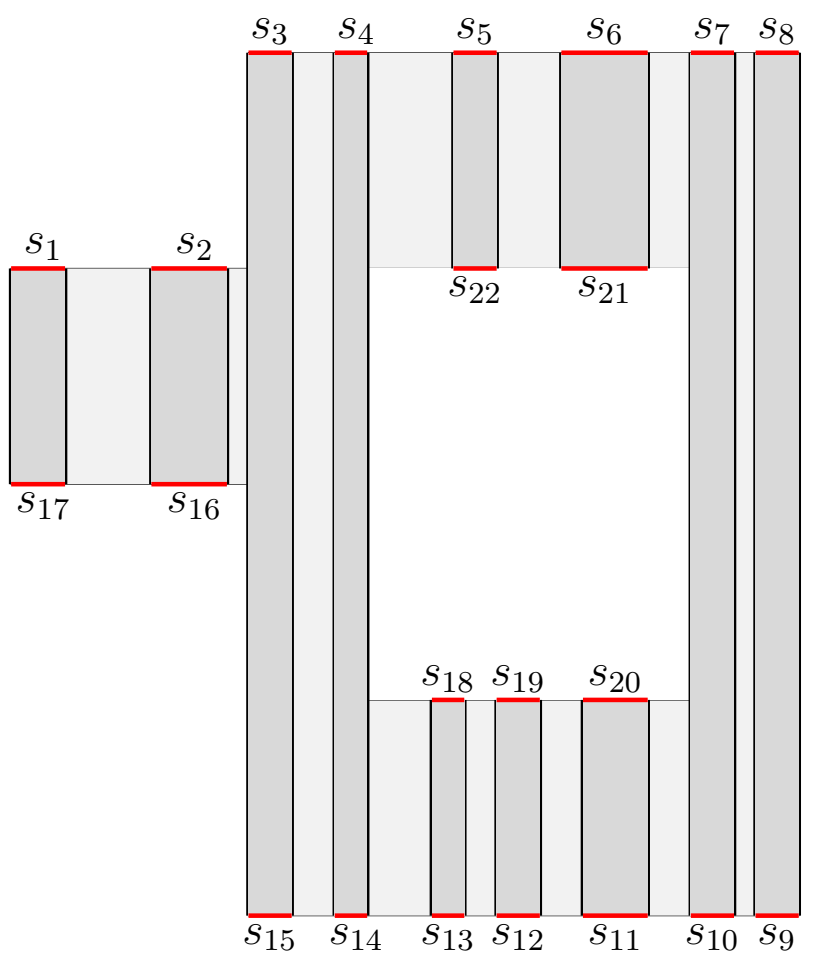

Fig. 6. The red segments $s_{j}$ (color online) for the bubble configuration of Fig. 4 in the deformed good region $G^{\prime}$. The segments can be naturally grouped in pairs: two segments form a pair if they belong to the boundary of the same droplet. E.g., $s_{1}$ and $s_{17}$ are paired, $s_{11}$ and $s_{20}$ are paired, etc. Every segment $s_{j}$ comes with its length $h\left(s_{j}\right)$, and with two spacings, the left spacing $w_{1}\left(s_{j}\right)$ and the right spacing $w_{2}\left(s_{j}\right)$, corresponding to the distances to the closest droplets to its left and to its right, by moving along the boundary. If one of the endpoints of $s_{j}$ is a corner of the boundary of $G^{\prime}$, then $s_{j}$ may not have any droplet to its left or right, in which case we let the corresponding spacing to be infinite. In the example in the figure, $w_{1}\left(s_{1}\right)=w_{1}\left(s_{17}\right)=w_{2}\left(s_{8}\right)=w_{2}\left(s_{9}\right)=+\infty$, and all the other spacings are finite

Lemma 3. Given $G^{\prime}$ and $\mathcal{B}_{G^{\prime}}$ as above, we have:

$$
E_{G^{\prime}}\left(\mathcal{B}_{G^{\prime}}\right) \geq \ell \sum_{j=1}^{N_{g}} e_{\infty}\left(h_{1}^{(j)}, w_{1}^{(j)}, \ldots, h_{n_{j}}^{(j)}\right)-\sum_{j=1}^{N_{s}} f\left(w_{1}\left(s_{j}\right), h\left(s_{j}\right), w_{2}\left(s_{j}\right)\right),
$$

where $h_{1}^{(j)}, w_{1}^{(j)}, \ldots, h_{n_{j}}^{(j)}$ is the sequence of widths and spacings associated to the slice $g_{j}$, and $e_{\infty}\left(h_{1}, w_{1}, \ldots, h_{n}\right)$ is the energy per unit vertical length of an infinite vertically striped configuration, with $n$ stripes of widths $h_{1}, \ldots, h_{n}$, separated among each other by spacings $w_{1}, \ldots, w_{n-1} \cdot{ }^{4}$ Moreover, $f\left(w_{1}, h, w_{2}\right)$ is half the interaction energy between the droplets in Fig. 7.

\footnotetext{
4 More precisely, $e_{\infty}\left(h_{1}, w_{1}, \ldots, h_{n}\right)$ is the energy per unit vertical length, measured with the Hamiltonian (1.1), of the infinite spin configuration on $\mathbb{Z}^{2}$, equal to -1 on the strips $\left\{\left(x_{1}, x_{2}\right) \in \mathbb{Z}^{2}: 0<x_{1}-\sum_{k=1}^{i-1}\left(h_{k}+\right.\right.$ $\left.\left.w_{k}\right) \leq h_{i}\right\}$, and +1 otherwise.
} 


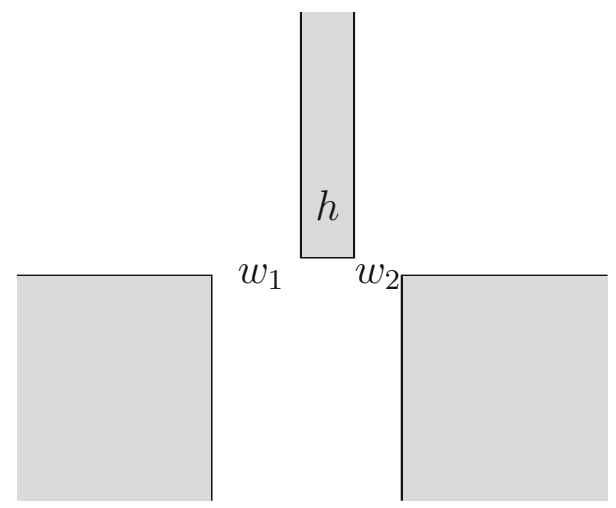

Fig. 7. A pictorial representation of the droplets involved in the definition of $f\left(w_{1}, h, w_{2}\right)$, which represents half the interaction energy of the infinite half strip of width $h$, with the two quarter spaces to its lower left and lower right. The spacings $w_{1}$ and $w_{2}$ represent the horizontal distances between the infinite half strip and the quarter space to its left and to its right, respectively

Remark. The second term on the right side of (2.17) represents the boundary error in the computation of the energy of the good region. Note that it scales proportionally to the boundary of $G^{\prime}$, rather than the total boundary of the slices $g_{j}$.

For future reference, it is useful to write in formulae the expressions of $e_{\infty}\left(h_{1}, w_{1}, \ldots\right.$, $\left.h_{n}\right)$ and $f\left(w_{1}, h, w_{2}\right)$. A straightforward computation shows that

$$
\begin{aligned}
e_{\infty}\left(h_{1}, w_{1}, \ldots, h_{n}\right)= & 4 J n-2 \sum_{i=1}^{n} \sum_{\mathbf{x} \in \mathbb{Z}^{2} \backslash\{\mathbf{0}\}} \frac{\min \left\{\left|x_{1}\right|, h_{i}\right\}}{|\mathbf{x}|^{p}} \\
& +\frac{1}{2} \sum_{\substack{i, j=1, \ldots, n \\
i \neq j}} W\left(l_{i}, \mathcal{L}\left(l_{j}\right)\right),
\end{aligned}
$$

where $l_{i}=\left\{(x, 0) \in \mathbb{Z}^{2}: 0<x-\sum_{k=1}^{i-1}\left(h_{k}+w_{k}\right) \leq h_{i}\right\}$ and $\mathcal{L}\left(l_{i}\right)$ is the smallest infinite vertical strip containing $l_{i}$, that is $\mathcal{L}\left(l_{i}\right)=\left\{(x, y) \in \mathbb{Z}^{2}: 0<x-\sum_{k=1}^{i-1}\left(h_{k}+w_{k}\right) \leq\right.$ $\left.h_{i}, y \in \mathbb{Z}\right\}$; moreover,

$$
f\left(w_{1}, h, w_{2}\right)=\frac{1}{2} W\left(\mathcal{L}_{h}^{+}, Q_{w_{1}, h, w_{2}}\right)
$$

where $\mathcal{L}_{h}^{+}=\left\{(x, y) \in \mathbb{Z}^{2}:-h \leq x<0, y>0\right\}$ and $Q_{w_{1}, h, w_{2}}=\left\{(x, y) \in \mathbb{Z}^{2}\right.$ : $x<-w_{1}-h$ or $\left.x \geq w_{2}, y \leq 0\right\}$.

Proof of Lemma 3. First of all, note that the contribution

$$
\sum_{\beta \in \mathcal{B}_{G^{\prime}}}\left[2 J\left|\Gamma_{\beta}\right|+u_{G^{\prime}}(\beta)\right]
$$

to the energy $E_{G^{\prime}}\left(\mathcal{B}_{G^{\prime}}\right)$ (see (2.8)) is identical to the corresponding contribution in $\ell \sum_{j=1}^{N_{g}} e_{\infty}\left(h_{1}^{(j)}, w_{1}^{(j)}, \ldots, h_{n_{j}}^{(j)}\right)$, i.e., to the one arising from the first two terms on the right side of (2.18). Therefore, all we have to prove is that the difference between the 
interaction terms in $E_{G^{\prime}}\left(\mathcal{B}_{G^{\prime}}\right)$ and in $\ell \sum_{j=1}^{N_{g}} e_{\infty}\left(h_{1}^{(j)}, w_{1}^{(j)}, \ldots, h_{n_{j}}^{(j)}\right)$ is bounded from below by the last sum in (2.17).

Let us focus on a given slice $g_{j}$ and on the intersection of a given bubble $\beta$ with this slice. The interaction energy of this portion of bubble with all the other bubbles in $G^{\prime}$, as it appears in $E_{G^{\prime}}\left(\mathcal{B}_{G^{\prime}}\right)$, is

$$
\frac{1}{2} \sum_{\beta^{\prime} \in \mathcal{B}_{G^{\prime}}}^{*} W\left(\delta_{\beta} \cap g_{j}, \delta_{\beta^{\prime}}\right)
$$

where we recall that the $*$ on the sum indicates the constraint that the bubbles $\beta^{\prime}$ intersecting $\beta$ after vertical translations should not be included in the sum. Equation (2.20) can be bounded from below by summing only over the bubbles $\beta^{\prime}$ having non zero intersection with $g_{j}$ :

$$
(2.20) \geq \frac{1}{2} \sum_{\substack{\beta^{\prime} \in \mathcal{B}_{G^{\prime}} \\ \beta^{\prime} \cap g_{j} \neq \emptyset, \beta^{\prime} \neq \beta}} W\left(\delta_{\beta} \cap g_{j}, \delta_{\beta^{\prime}}\right) .
$$

The term $\ell \sum_{j=1}^{N_{g}} e_{\infty}\left(h_{1}^{(j)}, w_{1}^{(j)}, \ldots, h_{n_{j}}^{(j)}\right)$ on the right side of (2.17) contains a term of that form, with the difference that $\delta_{\beta^{\prime}}$ is replaced by the infinite vertical strip of the same width containing it. In fact, the interaction term in (2.18) satisfies

$$
\ell \sum_{j=1}^{N_{g}} \sum_{\substack{i, i^{\prime}=1, \ldots, n_{j} \\ i \neq i^{\prime}}} W\left(l_{i}^{(j)}, \mathcal{L}\left(l_{i^{\prime}}^{(j)}\right)\right)=\sum_{j=1}^{N_{g}} \sum_{\beta \in \mathcal{B}_{G^{\prime}}} \sum_{\substack{\beta^{\prime} \in \mathcal{B}_{G^{\prime}} \\ \beta^{\prime} \cap g_{j} \neq \emptyset, \beta^{\prime} \neq \beta}} W\left(\delta_{\beta} \cap g_{j}, \mathcal{L}\left(\delta_{\beta^{\prime}}\right)\right),
$$

where $l_{i}^{(j)}$ is the analogue of the set $l_{i}$ defined after (2.18), corresponding to the widths and spacings $h_{1}^{(j)}, w_{1}^{(j)}, \ldots, h_{n_{j}}^{(j)}$ associated to the slice $g_{j}$. Therefore, what remains to be proved is that

$$
\sum_{j=1}^{N_{g}} \sum_{\beta \in \mathcal{B}_{G^{\prime}}} \sum_{\substack{\beta^{\prime} \in \mathcal{B}_{G^{\prime}} \\ \beta^{\prime} \cap g_{j} \neq \emptyset, \beta^{\prime} \neq \beta}} W\left(\delta_{\beta} \cap g_{j}, \mathcal{L}\left(\delta_{\beta^{\prime}}\right) \backslash \delta_{\beta^{\prime}}\right) \leq \sum_{j=1}^{N_{s}} W\left(\mathcal{L}_{h\left(s_{j}\right)}^{+}, Q_{w_{1}\left(s_{j}\right), h\left(s_{j}\right), w_{2}\left(s_{j}\right)}\right) .
$$

Let us rewrite the left side of (2.22) as

$$
\sum_{\beta^{\prime} \in \mathcal{B}_{G^{\prime}}} \sum_{\substack{j=1, \ldots, N_{g} \\ g_{j} \cap \beta^{\prime} \neq \emptyset \in \mathcal{B}_{G^{\prime}} \\ \beta \neq \beta^{\prime}}} W\left(\mathcal{L}\left(\delta_{\beta^{\prime}}\right) \backslash \delta_{\beta^{\prime}}, \delta_{\beta} \cap g_{j}\right) .
$$

Note that the horizontal boundary of $\mathcal{L}\left(\delta_{\beta^{\prime}}\right) \backslash \delta_{\beta^{\prime}}$ consists of two segments $s_{j}$, $s_{k}$ with $h\left(s_{j}\right)=h\left(s_{k}\right)$ and $s_{j}$ above $s_{k}$ (these are the pairs of segments mentioned in Sect. 2.4.2; see Fig. 6), and there is a one-to-one correspondence between summing over $\beta^{\prime}$ and summing over these pairs of segments $s_{j}, s_{k}$ (which is of course the same as summing 
over all segments). Moreover, the set $\mathcal{L}\left(\delta_{\beta^{\prime}}\right) \backslash \delta_{\beta^{\prime}}$ is equal to the union of two sets, each of which is a translation, and one also a reflection, of $\mathcal{L}_{h\left(s_{j}\right)}^{+}$, that is

$$
\mathcal{L}\left(\delta_{\beta^{\prime}}\right) \backslash \delta_{\beta^{\prime}}=\tau_{\beta^{\prime}}^{1} \mathcal{L}_{h\left(s_{j}\right)}^{+} \cup \tau_{\beta^{\prime}}^{2} r \mathcal{L}_{h\left(s_{j}\right)}^{+}
$$

for translations $\tau_{\beta^{\prime}}^{1}$ and $\tau_{\beta^{\prime}}^{2}$, and $r$ denoting reflection about the $x_{1}$-axis.

To conclude, it is enough to note that

$$
\bigcup_{\substack{i=1, \ldots, N_{g} \\ g_{i} \cap \beta^{\prime} \neq \emptyset}} \bigcup_{\beta \in \mathcal{B}_{G^{\prime}}} \delta_{\beta} \cap g_{i} \subseteq \tau_{\beta^{\prime}}^{1} Q_{w_{1}\left(s_{j}\right), h\left(s_{j}\right), w_{2}\left(s_{j}\right)} \cap \tau_{\beta^{\prime}}^{2} r Q_{w_{1}\left(s_{k}\right), h\left(s_{k}\right), w_{2}\left(s_{k}\right)},
$$

which implies the desired inequality (2.22), and thus completes the proof of Lemma 3.

2.4.3. Reflection Positivity. We now show how to bound from below the right side of (2.17), and how to use the resulting estimate to conclude the proof of Lemma 1. The key step is to use reflection positivity to obtain a lower bound on the $e_{\infty}$ term. This is an application of the block reflection positivity for one-dimensional spin systems worked out in [15]. The result is the following:

$$
e_{\infty}\left(h_{1}, w_{1}, \ldots, h_{n}\right) \geq \tau+\sum_{i=1}^{n} h_{i} e_{\mathrm{s}}\left(h_{i}\right)+\sum_{i=1}^{n-1} w_{i} e_{\mathrm{s}}\left(w_{i}\right)
$$

To see this, recall that $e_{\infty}\left(h_{1}, w_{1}, \ldots, h_{n}\right)$ is the energy per unit vertical length, measured with the Hamiltonian (1.1), of the infinite spin configuration on $\mathbb{Z}^{2}$, equal to -1 on the strips $\left\{\left(x_{1}, x_{2}\right) \in \mathbb{Z}^{2}: 0<x_{1}-\sum_{k=1}^{i-1}\left(h_{k}+w_{k}\right) \leq h_{i}\right\}$, and +1 otherwise. Since this spin configuration is quasi-one-dimensional (i.e., it is translation invariant in one of the two coordinate directions), its energy can be re-expressed in terms of a one-dimensional Hamiltonian, i.e., $e_{\infty}\left(h_{1}, w_{1}, \ldots, h_{n}\right)=\lim _{L \rightarrow \infty} H_{\Lambda_{L}}^{\text {per }}\left(\underline{\sigma}_{h_{1}, \ldots, h_{n}}\right)$, where:

1. Given a spin configuration $\underline{\sigma}_{\Lambda_{L}}$ on $\Lambda_{L}=[1, L] \cap \mathbb{Z}$,

$$
H_{\Lambda_{L}}^{\mathrm{per}}\left(\underline{\sigma}_{\Lambda_{L}}\right)=-J \sum_{i=1}^{L}\left(\sigma_{i} \sigma_{i+1}-1\right)+\sum_{1 \leq i<j \leq L}\left(\sigma_{i} \sigma_{j}-1\right) v_{L}(i-j),
$$

with

$$
v_{L}(x)=\sum_{n, y \in \mathbb{Z}}\left((x+n L)^{2}+y^{2}\right)^{-p / 2},
$$

and $\sigma_{L+1} \equiv \sigma_{1}$

2. The spins in the configuration $\underline{\sigma}_{h_{1}, \ldots, h_{n}}$ are equal to -1 on the intervals $\{x \in \mathbb{Z}$ : $\left.0<x-\sum_{k=1}^{i-1}\left(h_{k}+w_{k}\right) \leq h_{i}\right\}$, and +1 otherwise.

Now, $H_{\Lambda_{L}}^{\text {per }}\left(\underline{\sigma}_{h_{1}, \ldots, h_{n}}\right)$ is a one-dimensional spin Hamiltonian with a reflection positive long-range interaction and periodic boundary conditions, of the class considered in $[15,16]$. Therefore, we can apply the chessboard estimate proved e.g. in the Appendix of [16]. As a result, using [16, Eqs. (A4)-(A5)] and recalling the fact that the spin 
configuration $\underline{\sigma}_{h_{1}, \ldots, h_{n}}$ consists of blocks of alternating sign, of size $h_{1}, w_{1}, \ldots, h_{n}, w_{n}$, with $w_{n}=w_{n}(L) \stackrel{=}{=} L-\left(h_{1}+w_{1}+\cdots+h_{n}\right)$, we get

$$
H_{\Lambda_{L}}^{\mathrm{per}}\left(\underline{\sigma}_{\Lambda_{L}}\right) \geq \sum_{i=1}^{n}\left(h_{i} e_{\mathrm{S}}\left(h_{i}\right)+w_{i} e_{\mathrm{S}}\left(w_{i}\right)\right),
$$

where $e_{\mathrm{S}}(h)$ is the energy per site (as computed from $H_{\Lambda_{L}}^{\mathrm{per}}$, in the limit $L \rightarrow \infty$ ) of the infinite periodic configuration consisting of blocks all of the same size $h$, and of alternating sign. Note that $e_{\mathrm{S}}(h)$ is the same as the one defined in Sect. 1 for the two-dimensional model. Finally, to go from (2.29) to (2.26), observe that $\lim _{L \rightarrow \infty} w_{n}(L) e_{\mathrm{S}}\left(w_{n}(L)\right)=\tau$. This follows, e.g., from the explicit expression of $e_{\mathrm{S}}(h)$, derived in [20,21, Appendix A]:

$$
\begin{aligned}
e_{\mathrm{S}}(h) & =\frac{\tau}{h}+\frac{2}{h} \int_{0}^{\infty} \mathrm{d} \alpha \mu_{v}(\alpha) \frac{e^{-\alpha}}{\left(1-e^{-\alpha}\right)^{2}}\left(1-\tanh \frac{\alpha h}{2}\right) \\
& =\frac{\tau}{h}+\frac{A_{p}}{h^{p-2}}+O\left(h^{-p}\right)
\end{aligned}
$$

for large $h$, where $\mu_{v}(\alpha)$ is the inverse Laplace transform of the function $v_{\infty}(x)$ in (2.28), i.e., the function such that $v_{\infty}(x)=\int_{0}^{\infty} d \alpha \mu_{v}(\alpha) e^{-\alpha x}, \forall x>0$, and $A_{p}$ is a suitable constant.

Remark. From (2.30) it follows straight away that the optimal stripe width is $h^{*}=$ $\left((p-2) A_{p}|\tau|^{-1}\right)^{1 /(p-3)}(1+o(1))$ as $\tau \rightarrow 0$, and also that $e_{\mathrm{S}}\left(h^{*}\right)=\frac{p-3}{p-2} \frac{\tau}{h^{*}}(1+o(1))$.

2.4.4. Putting Things Together. Plugging (2.26) into (2.17) gives

$$
\begin{aligned}
E_{G^{\prime}}\left(\mathcal{B}_{G^{\prime}}\right) \geq & \left|G^{\prime}\right| e_{\mathrm{S}}\left(h^{*}\right)+\ell \tau N_{g}+\ell \sum_{j=1}^{N_{g}}\left[\sum_{k=1}^{n_{j}} h_{k}^{(j)}\left(e_{\mathrm{S}}\left(h_{k}^{(j)}\right)-e_{\mathrm{S}}\left(h^{*}\right)\right)\right. \\
& \left.+\sum_{k=1}^{n_{j}-1} w_{k}^{(j)}\left(e_{\mathrm{S}}\left(w_{k}^{(j)}\right)-e_{\mathrm{S}}\left(h^{*}\right)\right)\right]-\sum_{j=1}^{N_{s}} f\left(w_{1}\left(s_{j}\right), h\left(s_{j}\right), w_{2}\left(s_{j}\right)\right) .
\end{aligned}
$$

Now, $\ell N_{g} \leq \frac{1}{2}\left|\partial G^{\prime}\right|$ and it remains to show that the sum of the last two lines can be bounded from below by (const.) $\tau\left|\partial G^{\prime}\right|+\frac{1}{2} \sum_{h \neq h^{*}}\left(e_{\mathrm{S}}(h)-e_{\mathrm{S}}\left(h^{*}\right)\right) A_{h}(G)$. From the definition of $f\left(w_{1}, h, w_{2}\right)$ it easily follows that it can be bounded independently of $h$, as

$$
f\left(w_{1}, h, w_{2}\right) \leq \sum_{i=1,2} \frac{C_{2}}{w_{i}^{p-4}}
$$

for a suitable constant $C_{2}$. Moreover, from (2.30) it follows that

$$
e_{\mathrm{S}}(w)-e_{\mathrm{S}}\left(h^{*}\right) \geq \frac{C_{3}}{w^{p-2}}+\frac{\tau}{w}
$$


for all $w \geq 1$ and a suitable $C_{3}>0$. Note that the left side is non-negative, while the right side may be negative. A simple consequence of (2.33) is that

$$
\frac{C_{3}}{w^{p-4}} \leq|\tau| w+\left(C_{3}|\tau|^{-1}\right)^{\frac{1}{p-3}} w\left(e_{\mathrm{s}}(w)-e_{\mathrm{S}}\left(h^{*}\right)\right)
$$

In fact, if $w \leq\left(C_{3}|\tau|^{-1}\right)^{\frac{1}{p-3}}$, then (2.33) implies (2.34) directly. If, on the contrary, $w>\left(C_{3}|\tau|^{-1}\right)^{\frac{1}{p-3}}$, then $\frac{C_{3}}{w^{p-4}} \leq|\tau| w$, which is stronger than (2.34).

Using this bound in (2.32) implies that the last line of (2.31) can be bounded as

$$
\begin{aligned}
& \sum_{j=1}^{N_{s}} f\left(w_{1}\left(s_{j}\right), h\left(s_{j}\right), w_{2}\left(s_{j}\right)\right) \\
& \quad \leq \frac{C_{2}}{C_{3}} \sum_{j=1}^{N_{s}} \sum_{i=1,2}^{\prime}\left[w_{i}\left(s_{j}\right)|\tau|+\left(C_{3}|\tau|^{-1}\right)^{\frac{1}{p-3}} w_{i}\left(s_{j}\right)\left(e_{\mathrm{S}}\left(w_{i}\left(s_{j}\right)\right)-e_{\mathrm{S}}\left(h^{*}\right)\right)\right],
\end{aligned}
$$

where the prime on the sum indicates the constraint that $w_{i}\left(s_{j}\right)<\infty$. Now, every spacing $w_{i}\left(s_{j}\right)$ appears twice in the sum above (because every spacing is to the left or to the right of two different segments $\left.s_{j}, s_{j^{\prime}}\right)$, hence $\sum_{j=1}^{N_{s}} \sum_{i=1,2}^{\prime} w_{i}\left(s_{j}\right) \leq 2\left|\partial G^{\prime}\right|$. Similarly,

$$
\sum_{j=1}^{N_{s}} \sum_{i=1,2}^{\prime} w_{i}\left(s_{j}\right)\left(e_{\mathrm{s}}\left(w_{i}\left(s_{j}\right)\right)-e_{\mathrm{s}}\left(h^{*}\right)\right) \leq 2 \sum_{j=1}^{N_{g}} \sum_{k=1}^{n_{j}-1} w_{k}^{(j)}\left(e_{\mathrm{s}}\left(w_{k}^{(j)}\right)-e_{\mathrm{s}}\left(h^{*}\right)\right) .
$$

Therefore, if $\ell \geq 4 C_{2} C_{3}^{(4-p) /(p-3)}|\tau|^{-1 /(p-3)}$,

$$
\begin{aligned}
E_{G^{\prime}}\left(\mathcal{B}_{G^{\prime}}\right) \geq & \left|G^{\prime}\right| e_{\mathrm{S}}\left(h^{*}\right)+\tau\left|\partial G^{\prime}\right|\left(\frac{1}{2}+2 \frac{C_{2}}{C_{3}}\right) \\
& +\ell \sum_{j=1}^{N_{g}}\left[\sum_{k=1}^{n_{j}} h_{k}^{(j)}\left(e_{\mathrm{s}}\left(h_{k}^{(j)}\right)-e_{\mathrm{s}}\left(h^{*}\right)\right)+\frac{1}{2} \sum_{k=1}^{n_{j}-1} w_{k}^{(j)}\left(e_{\mathrm{s}}\left(w_{k}^{(j)}\right)-e_{\mathrm{s}}\left(h^{*}\right)\right)\right] .
\end{aligned}
$$

To complete the proof of Lemma 1 , note that $\left|G^{\prime}\right| \leq|G|$, and $\left|\partial G^{\prime}\right| \leq 2|\partial G|$, as already argued above.

Remark. The proof of (2.37) is valid for bubble configurations a bit more general than those considered here: in fact, we never used the fact that $\mathcal{B}_{G^{\prime}}$ has no holes, in the sense explained in Sect. 1.1.3. The only property we really used is that the bubbles in $\mathcal{B}_{G^{\prime}}$ are all rectangular with the same orientation.

2.5. Proof of Lemma 2. We proceed similarly to [20,21, Section 3]. The first step is to estimate the cost of erasing the bubbles with corners. Write $n_{c}(T)=\sum_{\beta \in \mathcal{B}_{T}} v_{c}(\beta)$, with $v_{c}(\beta)$ the number of corners associated with the bubble $\beta$, which may be an integer 
or a half-integer. Consider a bubble with $v_{c}(\beta)>0$. Dropping the positive interaction of this bubble with the others, its contribution to the energy is bounded from below as

$$
2 J\left|\Gamma_{\beta}\right|+u_{T}(\beta)+2^{1-p / 2} v_{c}(\beta) \geq \tau\left|\Gamma_{\beta}\right|+2^{1-\frac{p}{2}} v_{c}(\beta)
$$

Note that, in order for $\Gamma_{\beta}$ to be very long, the number of corners must be sufficiently large: in formulae (see [20,21, Eq. (3.10)] and following lines),

$$
\left|\Gamma_{\beta}\right| \leq 2 \ell+2 \ell v_{c}(\beta) \text {. }
$$

If, as we are assuming, $v_{c}(\beta) \geq 1 / 2$, then $v_{c}(\beta)+1 \leq 3 v_{c}(\beta)$, so that $v_{c}(\beta) \geq\left|\Gamma_{\beta}\right| /(6 \ell)$. Inserting this back into (2.38) gives

$$
\begin{aligned}
2 J\left|\Gamma_{\beta}\right|+u_{T}(\beta)+2^{1-p / 2} v_{c}(\beta) \geq & 2^{-p / 2} \frac{\left|\Gamma_{\beta}\right|}{6 \ell}\left(1-6 \cdot 2^{p / 2}|\tau| \ell\right) \\
& +2^{-\frac{p}{2}} v_{c}(\beta) .
\end{aligned}
$$

The first term on the right side is positive, and, therefore, can be dropped for a lower bound, if $\ell<\left(6 \cdot 2^{p / 2}|\tau|\right)^{-1}$. Therefore, denoting by $\mathcal{S}_{T}$ the subset of $\mathcal{B}_{T}$ consisting of all the bubbles without corners,

$$
E_{T}\left(\mathcal{B}_{T}\right) \geq E_{T}\left(\mathcal{S}_{T}\right)+2^{-\frac{p}{2}} n_{c}(T) .
$$

In order to estimate the energy of the corner-less configuration $\mathcal{S}_{T}$ we proceed exactly as in the proof Lemma 1. Assume that the contours in $\mathcal{S}_{T}$ are vertical. We deform the tile $T$ by moving to the right the left vertical boundary of $T$, until it hits the left vertical contour of a bubble, and vice versa for the right vertical boundary. We call $T^{\prime}$ and $\mathcal{S}_{T^{\prime}}$ the new region and configuration obtained after the deformation. In passing from $T, \mathcal{S}_{T}$ to $T^{\prime}, \mathcal{S}_{T^{\prime}}$ we increase the energy by at most $2|\tau| \ell$. Now we use the bound (2.37) which, as remarked after (2.37), is valid for all configurations consisting only of rectangular bubbles with the same orientation. The result is

$$
\begin{aligned}
E_{T^{\prime}}\left(\mathcal{S}_{T^{\prime}}\right) \geq & e_{\mathrm{S}}\left(h^{*}\right)\left|T^{\prime}\right|+C \tau \ell+\ell \sum_{i=1}^{n} h_{i}\left(e_{\mathrm{s}}\left(h_{i}\right)-e_{\mathrm{s}}\left(h^{*}\right)\right) \\
& +\frac{\ell}{2} \sum_{i=1}^{n-1} w_{i}\left(e_{\mathrm{S}}\left(w_{i}\right)-e_{\mathrm{S}}\left(h^{*}\right)\right),
\end{aligned}
$$

where $h_{1}, \ldots, h_{n}$ are the widths of the bubbles in $\mathcal{S}_{T^{\prime}}$, and $w_{1}, \ldots, w_{n-1}$ their separations.

It $T$ contains a hole, then either one of the $h_{i}$ 's or $w_{i}$ 's is larger than $\ell / 5$, or, the width of $T^{\prime}$ is smaller than $4 \ell / 5$. In the first case, one of the terms $\ell h_{i}\left(e_{\mathrm{s}}\left(h_{i}\right)-e_{\mathrm{S}}\left(h^{*}\right)\right)$ or $\ell w_{i}\left(e_{\mathrm{S}}\left(w_{i}\right)-e_{\mathrm{S}}\left(h^{*}\right)\right)$ is larger than $\left(\ell^{2} / 10\right)\left|e_{\mathrm{S}}\left(h^{*}\right)\right|$ : to see this recall that $\ell \geq c_{0} h^{*}$, for a large enough constant $c_{0}$, and use (2.30), which implies that $e_{\mathrm{S}}(h) \geq e_{\mathrm{S}}\left(h^{*}\right) / 2$ for $h \geq \ell / 5$. In the second case, the difference between $e_{\mathrm{S}}\left(h^{*}\right)\left|T^{\prime}\right|$ and $e_{\mathrm{S}}\left(h^{*}\right)|T|$ is larger than $\left(\ell^{2} / 5\right)\left|e_{\mathrm{S}}\left(h^{*}\right)\right|$. In both cases, we get a gain of at least $\left(\ell^{2} / 20\right)\left|e_{\mathrm{S}}\left(h^{*}\right)\right|$, which is larger than (const.) $\ell^{2}|\tau|^{(p-2) /(p-3)}$. To conclude the proof, note that under the stated assumptions on $\ell$ (that is, $c_{0} h^{*} \leq \ell \leq\left(c_{0}|\tau|\right)^{-1}$ for a suitable constant $c_{0}$ ), the error term $(2+C)|\tau| \ell$ is smaller than $c\left[n_{c}(T)+|\tau|^{(p-2) /(p-3)} \ell^{2} \chi_{\text {hole }}(T)\right]$, where $c$ can be made as small as desired, by increasing $c_{0}$ (recall also that by definition of bad tile, either $n_{c}(T) \geq 1 / 2$, or $\left.\chi_{\text {hole }}(T)=1\right)$. This concludes the proof. 
Acknowledgments. Open access funding provided by Institute of Science and Technology (IST Austria). The research leading to these results has received funding from the European Research Council under the European Union's Seventh Framework Programme ERC Starting Grant CoMBoS (Grant Agreement No. 239694), from the Italian PRIN National Grant Geometric and analytic theory of Hamiltonian systems in finite and infinite dimensions, and the Austrian Science Fund (FWF), project Nr. P 27533-N27. Part of this work was completed during a stay at the Erwin Schrödinger Institute for Mathematical Physics in Vienna (ESI program 2015 "Quantum many-body systems, random matrices, and disorder"), whose hospitality and financial support is gratefully acknowledged. We also thank Joel Lebowitz and Elliott Lieb for stimulating discussions and their constant encouragement in pursuing this project.

Open Access This article is distributed under the terms of the Creative Commons Attribution 4.0 International License (http://creativecommons.org/licenses/by/4.0/), which permits unrestricted use, distribution, and reproduction in any medium, provided you give appropriate credit to the original author(s) and the source, provide a link to the Creative Commons license, and indicate if changes were made.

\section{A. The Higher-Dimensional Case}

In this appendix we shall detail our main results in the case $d \geq 3$, and explain the main differences in their proof as compared to the two-dimensional case. The starting point is a representation of the energy in terms of droplets as in (2.3), whose boundaries, separating plus spins from minus spins, consist now of $d-1$ dimensional plaquettes. Tiles are now $d$-dimensional cubes of side length $\ell$, and are used to divide space into good regions and bad cubes, with the good regions only containing "stripes" (i.e., quasione-dimensional regions of uniform spins, delimited by two flat parallel interfaces; they are slabs in $d=3$ ), which can be oriented in $d$ different directions.

Our first claim concerns the fact that the localization bound (2.6) still holds, with the obvious notion of "corner", namely $d-2$ dimensional segments where two plaquettes with different orientation meet. The proof of the analogue of (2.6) in higher dimensions is essentially the same as in $d=2$, and relies on the analogue of (2.10), whose proof is in [20,21, App. D].

The key bound in Lemma 1 for the good regions holds verbatim also for general $d \geq 2$. After the modification from $G$ to $G^{\prime}$, each stripe has a definite width $h$ but will not be a cuboid, in general; it is bounded by a union of $d-1$ dimensional cuboids $s_{k}$ with width $h$ and all other dimensions equal to $\ell$. As before, each $s_{k}$ comes with two numbers, $w_{1}\left(s_{k}\right)$ and $w_{2}\left(s_{k}\right)$, measuring the distance to the next slice in the direction perpendicular to the stripes. The analogues of the slices $g_{j}$ introduced in Sect. 2.4.2 are cylinders with base area $\ell^{d-1}$ and various heights, which are obtained by adding up the various stripe widths $h_{i}$ and their separation $w_{i}$; they are oriented perpendicular to the stripes. With these modified definitions, Eq. (2.17) still holds, with $\ell$ replaced by $\ell^{d-1}$ in front of the first term on the right side, and $f\left(w_{1}, h, w_{2}\right)$ now denoting the interaction energy as depicted in Fig. 7, with the upper strip of width $h$ extended by $\ell$ in the remaining $d-2$ dimensions, while the two lower ones are infinite in those directions. This function $f$ satisfies the bound

$$
f\left(w_{1}, h, w_{2}\right) \leq \ell^{d-2} \sum_{i=1,2} \frac{C_{2}}{w_{i}^{p-d-2}} .
$$

As already discussed in [20,21, App. A], the analogue of (2.30) for general $d$ is

$$
e_{\mathrm{s}}(h)=\frac{\tau}{h}+\frac{A_{p, d}}{h^{p-d}}+O\left(h^{d-p-2}\right)
$$


from which it follows that $e_{\mathrm{S}}\left(h^{*}\right) \sim|\tau|^{(p-d) /(p-d-1)}$ and $h^{*} \sim|\tau|^{-1 /(p-d-1)}$ for small $\tau$. Moreover, one easily deduces that (A.1) can be bounded by

$$
\frac{C_{3}}{w^{p-d-2}} \leq|\tau| w+\left(C_{3}|\tau|^{-1}\right)^{\frac{1}{p-d-1}} w\left(e_{\mathrm{S}}(w)-e_{\mathrm{S}}\left(h^{*}\right)\right)
$$

which is the analogue of Eq. (2.34). The rest of the proof of Eq. (2.13) for general $d$ remains unchanged.

The analogue of Lemma 2 for general $d \geq 2$ takes the following form:

Lemma 2' For given $d \geq 2$, there exist positive constants $c_{0}, c_{2}$ and $\varepsilon$ such that, if $-\varepsilon<\tau<0$ and $c_{0} h^{*} \leq \bar{\ell} \leq\left(c_{0}|\tau|\right)^{-1 /(d-1)}$, then the energy $E_{T}$ of any bad tile $T \in \mathcal{P}$ satisfies

$$
E_{T}\left(\mathcal{B}_{T}\right) \geq \ell^{d} e_{\mathrm{s}}\left(h^{*}\right)+c_{2}\left[n_{c}(T)+|\tau|^{(p-d) /(p-d-1)} \ell^{d} \chi_{\text {hole }}(T)\right],
$$

where $\chi_{\text {hole }}(T)$ is equal to 1 if $T$ contains a hole, and 0 otherwise.

Its proof is a rather straightforward adaptation of the one in $d=2$, and we refer to [20,21, App. D], where the necessary changes were described in the case $d=3$.

Since every portion of the boundary of a good region $G_{i}$ is adjacent to a bad tile, we have the bound $\sum_{i=1}^{\mathcal{N}_{G}}\left|\partial G_{i}\right| \leq 2 d \ell^{d-1} \mathcal{N}_{B}$. In combination with the bounds above, this leads to the following generalization of Theorem 3 .

Theorem 3' For given $d \geq 2$, there exist positive constants $C_{0}, C_{1}, \varepsilon$ such that, if $J_{c}-\varepsilon<J<J_{c}$ and $C_{0} h^{*} \leq \ell \leq\left(C_{0}\left(J_{c}-J\right)\right)^{-1 /(d-1)}$, then for every $\underline{s} \in\{ \pm 1\}^{\mathbb{Z}^{d}}$ and every finite set $X \subset \mathbb{Z}^{d}$,

$$
\begin{aligned}
H_{X}\left(\underline{s}_{X} \mid \underline{\sigma}^{*}\right) \geq & H_{X}\left(\underline{\sigma}_{X}^{*} \mid \underline{\sigma}^{*}\right)+C_{1}\left(N_{c}+\left(J_{c}-J\right)^{\frac{p-d}{p-d-1}} \ell^{d} \mathcal{N}_{B}^{\text {hole }}\right) \\
& +\frac{1}{2} \sum_{h \neq h^{*}} \sum_{i=1}^{\mathcal{N}_{G}}\left(e_{\mathrm{S}}(h)-e_{\mathrm{S}}\left(h^{*}\right)\right) A_{h}\left(G_{i}\right),
\end{aligned}
$$

where $N_{c}, \mathcal{N}_{B}^{\text {hole }}$, and $G_{i}$ are, respectively, the number of corners, the number of bad tiles containing a hole, and the good regions, associated with the infinite spin configuration $\underline{\sigma}=\left(\underline{s}_{X}, \underline{\sigma}_{X^{c}}^{*}\right)$ coinciding with $\underline{s}_{X}$ on $X$ and with $\underline{\sigma}^{*}$ on $X^{c}$, defined via tiling with squares of side length $\ell$ as described above.

Theorem 3' implies the analogue of Theorem 1, i.e., the fact that striped configurations with stripe width $h^{*}$ are infinite volume ground states with trivial sectors for $J$ close to $J_{c}$, and also the analogue of Theorem 2 , stating that all infinite volume ground states that are invariant under translations by $d-1$ lattice vectors are characterized by the existence of an interface separating the cubic lattice $\mathbb{Z}^{d}$ into two components, on each of which the configuration is perfectly striped.

\section{References}

1. Arlett, J., Whitehead, J.P., MacIsaac, A.B., De'Bell, K.: Phase diagram for the striped phase in the two-dimensional dipolar Ising model. Phys. Rev. B 54, 3394 (1996)

2. Ball, P.: The Self-Made Tapestry: Pattern Formation in Nature. Oxford University Press, Oxford (1999)

3. Biskup, M., Chayes, L., Kivelson, S.A.: On the absence of ferromagnetism in typical 2D ferromagnets. Commun. Math. Phys. 274, 217-231 (2007) 
4. Cannas, S.A., Michelon, M.F., Stariolo, D.A., Tamarit, F.A.: Ising nematic phase in ultrathin magnetic films: a Monte Carlo study. Phys. Rev. B 73, 184425 (2006)

5. Chakrabarty, S., Dobrosavljevic, V., Seidel, A., Nussinov, Z.: Universality of modulation length and time exponents. Phys. Rev. E 86, 041132 (2012)

6. Chakrabarty, S., Nussinov, Z.: Modulation and correlation lengths in systems with competing interactions. Phys. Rev. B 84, 144402 (2011)

7. Chayes, L., Emery, V., Kivelson, S., Nussinov, Z., Tarjus, G.: Avoided critical behavior in a uniformly frustrated system. Phys. A 225, 129 (1996)

8. Cinti, F., Portmann, O., Pescia, D., Vindigni, A.: One-dimensional Ising ferromagnet frustrated by longrange interactions at finite temperatures. Phys. Rev. B 79, 214434 (2009)

9. Czech, R., Villain, J.: Instability of two-dimensional Ising ferromagnets with dipole interactions. J. Phys. Condens. Matter 1, 619 (1989)

10. DeBell, K., MacIsaac, A.B., Whitehead, J.P.: Dipolar effects in magnetic thin films and quasi-twodimensional systems. Rev. Mod. Phys. 72, 225 (2000)

11. Edlund, E., Nilsson Jacobi, M.: Universality of striped morphologies. Phys. Rev. Lett. 105, 137203 (2010)

12. Emery, V.J., Kivelson, S.A., Tranquada, J.M.: Stripe phases in high-temperature superconductors. Proc. Natl. Acad. Sci. USA 96, 8814-8817 (1999)

13. Flatley, L.C., Theil, F.: Face-centered cubic crystallization of atomistic configurations. Arch. Ration. Mech. Anal. 218, 363-416 (2015)

14. Fradkin, E., Kivelson, S.A.: Liquid-crystal phases of quantum Hall systems. Phys. Rev. B 59, 8065 (1999)

15. Giuliani, A., Lebowitz, J., Lieb, E.: Ising models with long-range dipolar and short range ferromagnetic interactions. Phys. Rev. B 74, 064420 (2006)

16. Giuliani, A., Lebowitz, J., Lieb, E.: Striped phases in two-dimensional dipole systems. Phys. Rev. B 76, 184426 (2007)

17. Giuliani, A., Lebowitz, J., Lieb, E.: Periodic minimizers in 1D local mean field theory. Commun. Math. Phys. 286, 163177 (2009)

18. Giuliani, A., Lebowitz, J., Lieb, E.: Modulated phases of a 1D sharp interface model in a magnetic field. Phys. Rev. B 80, 134420 (2009)

19. Giuliani, A., Lebowitz, J., Lieb, E.: Checkerboards, stripes and corner energies in spin models with competing interactions. Phys. Rev. B 84, 064205 (2011)

20. Giuliani, A., Lieb, E.H., Seiringer, R.: Formation of stripes and slabs near the ferromagnetic transition. Comm. Math. Phys. 331, 333-350 (2014)

21. Giuliani, A., Lieb, E.H., Seiringer, R.: Realization of stripes and slabs in two and three dimensions. Phys. Rev. B 88, 064401 (2013)

22. Giuliani, A., Müller, S.: Striped periodic minimizers of a two-dimensional model for martensitic phase transitions. Commun. Math. Phys. 309, 313-339 (2012)

23. Grousson, M., Tarjus, G., Viot, P.: Phase diagram of an Ising model with long-range frustrating interactions: A theoretical analysis. Phys. Rev. E 62, 7781 (2000)

24. Harrison, C. et al.: Mechanisms of ordering in striped patterns. Science 24, 1558 (2000)

25. Heitmann, R.C., Radin, C.: The ground state for sticky disks. J. Stat. Phys. 22, 281 (1980)

26. Kennedy, T., Lieb, E.H.: An itinerant electron model with crystalline or magnetic long range order. Phys. A 138, $320(1986)$

27. Kohn, R.V., Müller, S.: Branching of twins near an austenite-twinned-martensite interface. Philos. Mag. A 66, 697 (1992)

28. Low, U., Emery, V.J., Fabricius, K., Kivelson, S.A.: Study of an Ising model with competing long- and short-range interactions. Phys. Rev. Lett. 72, 1918 (1994)

29. MacIsaac, A.B., Whitehead, J.P., Robinson, M.C., DeBell, K.: Striped phases in two-dimensional dipolar ferromagnets. Phys. Rev. B 51, 16033 (1995)

30. Mendoza-Coto, A., Stariolo, D.A., Nicolao, L.: Nature of long range order in stripe forming systems with long range repulsive interactions. Phys. Rev. Lett. 114, 116101 (2015)

31. Nielsen, E., Bhatt, R.N., Huse, D.A.: Modulated phases in magnetic models frustrated by long-range interactions. Phys. Rev. B 77, 054432 (2008)

32. Okamoto, M., Maruyama, T., Yabana, K., Tatsumi, T.: Nuclear "pasta” structures in low-density nuclear matter and properties of the neutron-star crust. Phys. Rev. C 88, 025801 (2013)

33. Osenda, O., Tamarit, F.A., Cannas, S.A.: Nonequilibrium structures and slow dynamics in a two-dimensional spin system with competing long-range and short-range interactions. Phys. Rev. E 80, 021114 (2009)

34. Pighin, S.A., Cannas, S.A.: Phase diagram of an Ising model for ultrathin magnetic films: comparing mean field and Monte Carlo predictions. Phys. Rev. B 75, 224433 (2007)

35. Portmann, O., Golzer, A., Saratz, N., Billoni, O.V., Pescia, D., Vindigni, A.: Scaling hypothesis for modulated systems. Phys. Rev. B 82, 184409 (2010) 
36. Rastelli, E., Regina, S., Tassi, A.: Phase transitions in a square Ising model with exchange and dipole interactions. Phys. Rev. B 73, 144418 (2006)

37. Seul, M., Wolf, R.: Evolution of disorder in two-dimensional stripe patterns: "Smectic" instabilities and disclination unbinding. Phys. Rev. Lett. 68, 2460 (1992)

38. Spivak, B., Kivelson, S.A.: Phases intermediate between a two-dimensional electron liquid and Wigner crystal. Phys. Rev. B 70, 155114 (2004)

39. Stoycheva, A.D., Singer, S.J.: Stripe melting in a two-dimensional system with competing interactions. Phys. Rev. Lett. 84, 4657 (2000)

40. Süto, A.: Crystalline ground states for classical particles. Phys. Rev. Lett. 95, 265501 (2005)

41. Theil, F.: A Proof of crystallization in two dimensions. Commun. Math. Phys. 262, 209 (2006)

42. Vindigni, A., Saratz, N., Portmann, O., Pescia, D., Politi, P.: Stripe width and nonlocal domain walls in the two-dimensional dipolar frustrated Ising ferromagnet. Phys. Rev. B 77, 092414 (2008)

Communicated by L. Erdös 